\title{
SHAPES OF EPITAXIALLY GROWN QUANTUM DOTS
}

\author{
IRENE FONSECA, ALDO PRATELLI AND BARBARA ZWICKNAGL
}

\begin{abstract}
A variational model introduced by Spencer and Tersoff (Appl. Phys. Lett. 96:073114, 2010) to describe optimal faceted shapes of epitaxially deposited films is studied analytically in the case in which there are a non-vanishing crystallographic miscut and a lattice incompatibility between the film and the substrate. Existence of faceted minimizers for every volume of the deposited film is established. In particular, it is shown that there is no wetting effect for small volumes. Geometric properties including a faceted version of the zero contact angle are derived, and the explicit shapes of minimizers for small volumes are identified.
\end{abstract}

\section{Contents}

1. Introduction 1

2. Notation and technical results 5

2.1. Notation 5

2.2. The "Killing Lemma" 6

2.3. A bound on the length $W$

3. Relaxation of the energy 20

4. Theorem A: existence of classical minimizers 27

5. Estimates for rescaled profiles 29

6. Theorem B: generalized zero contact angle 31

7. Theorem C: the minimizers for small volumes are half-pyramids 35

Acknowledgements $\quad 38$

References $\quad 38$

\section{INTRODUCTION}

In this paper we study analytically a variational model introduced by Spencer and Tersoff (see $[18,22,17])$ to describe self-organized surface morphologies in epitaxially strained crystalline fims. If a thin elastic film is deposited epitaxially on a rigid substrate, then the mismatch between the two lattices induces a strain near the interface. The competition between the strain energy and the surface energy of the film's free surface drives the formation of different island morphologies depending on the volume of the film and the amplitude of the crystallographic misfit (see, e.g., $[2,10,22,15,21])$. These self-assembled quantum-dots have proven to be useful

2000 Mathematics Subject Classification. 74G65, 74K35, 49Q20, 49K05.

Key words and phrases. epitaxially strained elastic films, shape formation, miscut, relaxation, existence of minimizers. 
in many technological applications, including optical and optoelectronic devices like quantum dot lasers (see [20] and the references therein).

We study a fully faceted model as developed in $[18,22]$, which takes into account in particular the miscut angle between the substrate and the film as has been observed experimentally, e.g., in the growth of Germanium $(\mathrm{Ge})$ on Silicon $(\mathrm{Si})$ substrates (see [21]). In particular, a non-zero miscut angle favours asymmetric island shapes (see $[21,14,5]$ ) which could be useful in optical applications (see [18]).

The energy functional under consideration consists of the strain energy stored in the film and the interfacial energy of its free, faceted surface. We restrict ourselves to two-dimensional structures, which correspond to three-dimensional islands with planar symmetry. This is done for simplicity in several works on the subject (see e.g. [18, 22, 7, 9]). We refer to [4] and [6] (see also [11]) for some results on related problems in higher dimensions. Further, we consider here the stationary problem; some results for related evolutionary problems in this context have been recently obtained (see $[8,13,6]$ ).

A profile $S(u)$ is described as the subgraph of a height profile function $u: \mathbb{R} \rightarrow[0, \infty)$ (see Figure 1), i.e.,

$$
S(u):=\left\{(x, y) \in \mathbb{R}^{2}: 0 \leq y \leq u(x)\right\},
$$

where $u$ denotes the height with respect to the substrate orientation. A function $u \in W^{1, \infty}(\mathbb{R})$ corresponds to a "classically admissible" faceted profile if $u \geq 0$ and, for almost every $x$ in the support of $u, u^{\prime}(x)=\tan \alpha$ for some $\alpha=\alpha(x)=-\theta_{m}+n \theta$, where $\theta>\theta_{m}>0$ are given angles, the latter being called miscut angle, and where $n=n(x)$ is an integer such that $-\pi / 2<\alpha<\pi / 2$. For instance, typical values of $\theta$ and the miscut angle $\theta_{m}$ are $\theta_{m} \approx 3^{\circ}$ and $\theta \approx 11.3^{\circ}$, which for $n=3$ correspond roughly to $\{105\},\{113\}$ and $\{111\}$ facets on (001), see [18] for a discussion. Observe that, for these values of $\theta_{m}$ and $\theta$, the admissible values for the integer $n$ could be $-7 \leq n \leq 8$; nevertheless, one usually takes into account only $n$ with smaller values of $|n|$ because for large angles the expressions for the energy given below become too inaccurate, for instance in [19] the authors consider only $-3 \leq n \leq 3$. For our mathematical analysis, the choice of the admissible numbers $n$ does not make any difference.

Experiments and numerical simulations suggest that the profile $S(u)$ is made of a more or less periodic array of "islands", i.e., connected components of the set $\{u>0\}$, and thus, we are led to study a single island. Precisely, in what follows we will assume that $\{u>0\}=(0, W)$, with $W>0$ depending on the function $u$. Consequently, we identify an admissible profile function $u$ with its restriction to $[0, W]$, and in particular use the notation

$$
\operatorname{length}(\operatorname{Graph}(u)):=\operatorname{length}\left(\operatorname{Graph}\left(\left.u\right|_{[0, W]}\right)\right)=\int_{0}^{W} \sqrt{1+\left|u^{\prime}(x)\right|^{2}} d x .
$$

The formation of the island $S(u)$ requires a total energy

$$
E(u):=E(S(u)):=E_{\mathrm{S}}(u)+E_{\mathrm{r}}(u),
$$


where the elastic (or strain) energy is

$$
E_{\mathrm{r}}(u):=S_{0} \frac{2}{\pi} \int_{0}^{W} \int_{0}^{W} u^{\prime}(x) u^{\prime}(y) \ln |x-y| d y d x
$$

and the extra surface energy (that we will call for brevity just surface energy) is

$$
E_{\mathrm{S}}(u):=\gamma_{0} \operatorname{length}(\operatorname{Graph}(u))-\gamma_{m} W,
$$

where $\gamma_{0}$ and $\gamma_{m}$ are the free surface energy densities and the contact surface energy density, respectively. As it is customary, in this paper we will restrict to the case in which $\gamma_{0}=\gamma_{m}$ (e.g., see $[18])$.

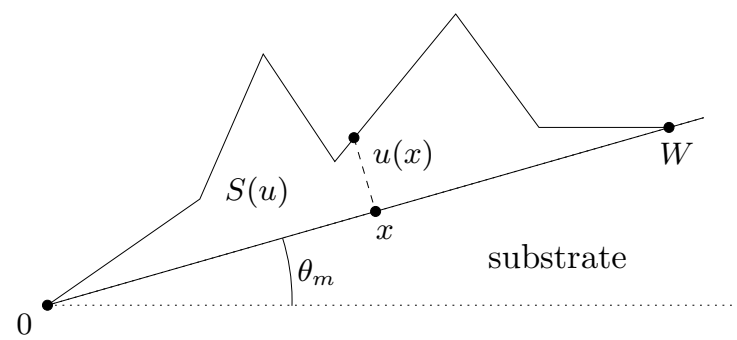

FIgURE 1. Sketch of a faceted height profile function $u$ with support $[0, W]$. The profile is Lipschitz and the derivative lies almost everywhere in a discrete set. The miscut angle is denoted by $\theta_{m} \neq 0$, i.e., the preferred orientation of the film is not parallel to the substrate surface.

Mathematical studies of the formation of quantum dots have been mostly devoted to continuum models for the strain energy in the geometrically linear approximation of small strain elasticity (see $[16,7,9,2,11,1]$ ). The strain energy (1.2) arises as small slope approximation of a geometrically linear elastic strain energy (see [22]). The goal of this paper is to rigorously analyze the model and validate some geometric properties that have been found experimentally or numerically (see $[18,2]$ ). For this purpose, we study minimizers of the total energy (1.1) under a volume or mass constraint

$$
\int_{0}^{W} u(x) d x=m
$$

Before highlighting some of our main results, and since there are in the literature many different models to study the behaviour of elastic thin films, it is convenient to underline some of the differences between these models and the present one. Keep in mind that the main peculiarities of the present model are that

a) we consider a faceted case (i.e., only a finite number of slopes are admissible), and

b) we explicitly take in account a non-zero miscut angle $\theta_{m}$.

A typical phenomenon which arises with most of the different models is the so-called wetting (see for instance $[9,7,1]$ ), i.e., optimal profiles tend to be extremely large and flat when the mass (or, equivalently, the volume) tends to 0 . In this case, one observes that $W \rightarrow \infty$ and $u \approx$ const. $\ll 1$ for the optimal profiles $u$, and the main property of the wetting solutions is 
that the surface energy vanishes in the limit $W \rightarrow \infty$. However, notice that with our model the wetting is impossible, because flat profiles are not admissible.

Another common property of solutions is the "zero contact angle" (see [7, 9, 1]), namely, the fact that islands always meet the substrate tangentially. Also this property is impossible in our context, since only a finite number of slopes are admissible.

In models similar to the present one, but without the constraint of faceted profiles, much attention has been devoted to regularity results of optimal profile functions (see $[3,7,9,1]$ ). However, note that in the present setting, any non-trivial classically admissible profile function cannot be any smoother than $W^{1, \infty}$.

We now discuss features of the energy functional (1.1). It is important to observe that, as is typical for pattern formation models, the behaviours of the elastic and surface energies are completely different. In fact, the convexity of the surface energy suggests that an optimal profile should be as flat as possible but, on the other hand, the concavity of the logarithm pushes toward less simple profiles. Moreover, the scaling of the surface energy is linear, while the scaling of the elastic energy is quadratic (see Section 5). The formation of islands on the substrate surface is then the result of the competition between these two terms, and a consequence of their different scaling is that the qualitative shape of the optimal profile changes as the volume $|S(u)|$ of the film increases (see $[2,10,12,18,21,11,1]$ ). In particular, if the volume is small then the surface energy dominates and the film forms an as flat as possible layer (this is the more or less heuristic reason why the wetting phenomenon described above occurs). As observed before, the presence of a miscut angle implies that this "as flat as possible" profile is not the infinitely wide and flat one; instead, it is simple to guess that this flattest profile is nothing but the half-pyramid, shown in Figure 2 (see [18]). Indeed, in Section 7 we give a rigorous proof of the fact that, for small volume, the solution is exactly the half-pyramid. This is a sort of "faceted wetting effect", and this effect was already numerically foreseen and experimentally observed, see [18, 19].

We will be able to establish also a faceted analogue to the "zero-contact angle" effect, that is, the islands meet the substrate not tangentially, but at the minimal admissible slope (see Section 6). Notice that, according to our notation, this minimal admissible slope is $\theta-\theta_{m}>0$ in the left contact point, and $-\theta_{m}<0$ in the right contact point. Observe also that the latter angle is horizontal in our pictures (e.g., refer to Figure 1).

The remainder of the manuscript is organized as follows. In Section 2, we collect the notation and some technical results that are used throughout the paper. This includes a bound on the length $W$ of low energy island shapes (see Proposition 2.4). Precisely, we show that for fixed mass $m$, the energy explodes as $W \rightarrow \infty$. In particular, for low-energy sequences, there is a uniform bound on the supports. This tightness is the main ingredient to derive compactness of minimizing sequences.

In Section 3, the relaxation of the energy is derived (see Theorem 3.5). It turns out that the relaxation $\bar{E}$ of the total energy $\bar{E}$ is decoupled and given by $\overline{E_{\mathrm{S}}}+\overline{E_{\mathrm{r}}}$, where $\overline{E_{\mathrm{r}}}$ and $\overline{E_{\mathrm{S}}}$ denote the relaxations of the strain and the surface energies, respectively, and moreover $\bar{E}(u)=E(u)$ for all classical $u$. 


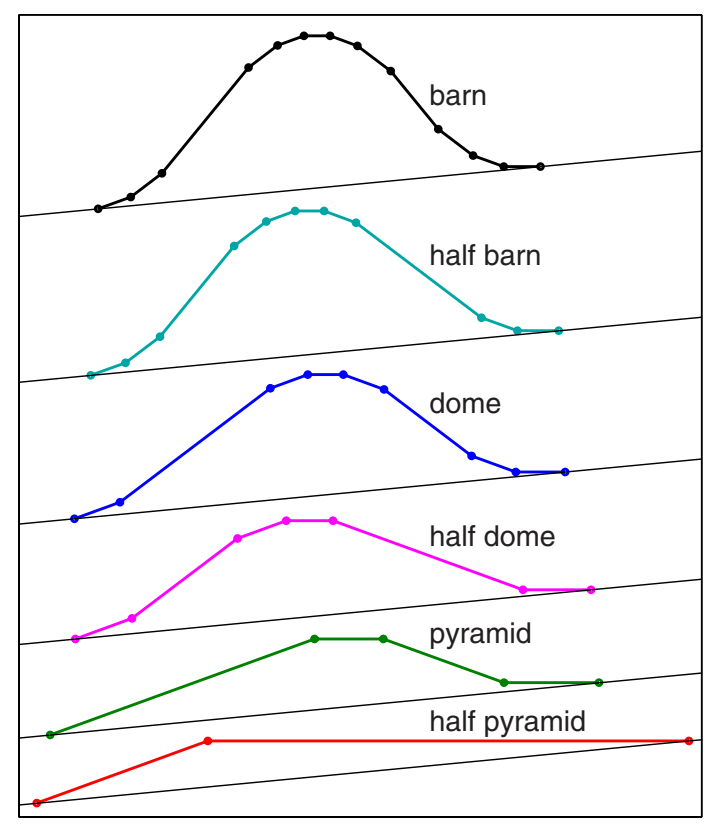

FIgURE 2. Shape transitions with increasing volume at miscut angle $3^{\circ}$. Numerical simulation. Courtesy of B. Spencer and J. Tersoff. Reproduced with permission from Figure 1 of "Asymmetry and shape transitions of epitaxially strained islands on vicinal surfaces" by B. J. Spencer and J. Tersoff, Appl. Phys. Lett. 96/7, 073114 (2010). Copyright 2010, AIP Publishing LLC.

Subsequently, we prove that there exist minimizers of the relaxed energy for any given volume, and that any such minimizer $u_{*}$ is classically admissible, i.e., faceted, that is $u_{*}^{\prime}(x)=$ $-\theta_{m}+n \theta$ almost everywhere (see Theorem $\mathrm{A}$ in Section 4).

The remaining part of the paper is devoted to proving geometric properties of minimizers. Main tools in the proofs are variations of a "killing strategy" as illustrated in Lemma 2.1 and the analysis of rescaled profiles undertaken in Section 5. Further, we establish a faceted analogue to the zero-contact angle property, namely that the island meets the substrate at minimal admissible slope (see Theorem B in Section 6). Finally, we show that for small volumes, the minimizers of the energy are half-pyramids (see Theorem $\mathrm{C}$ in Section 7 ).

\section{Notation AND technical RESUlts}

2.1. Notation. As detailed in the Introduction, we focus on the case in which the surface energy density constants $\gamma_{0}$ and $\gamma_{m}$ coincide, $\gamma_{0}=\gamma_{m}$, and where there is a non-trivial miscut angle $\theta_{m}>0$. We consider the set $\mathcal{N}:=\left\{n \in \mathbb{Z}, n^{-} \leq n \leq n^{+}\right\}$, where $n^{-}<0<n^{+}$are two integers such that

$$
-\frac{\pi}{2}<n^{-} \theta-\theta_{m}<0<n^{+} \theta-\theta_{m}<\frac{\pi}{2},
$$


and where $\theta$ is another fixed material parameter. We recall that the only admissible slopes for admissible profiles $u$ are $-\theta_{m}+n \theta$, for $n \in \mathcal{N}$. For the standard case $\left(\theta \approx 11.3^{\circ}\right.$ and $\left.\theta_{m} \approx 3^{\circ}\right)$ the largest possible set $\mathcal{N}$ is $\{-7 \leq n \leq 8\}$, but one can consider, for physical reasons, a smaller choice, such as $\{-3 \leq n \leq 3\}$, as in [19]. These restrictions do not make any substantial difference to our analysis, and, accordingly, the set $\mathcal{A}$ of all classically admissible functions is given by

$$
\mathcal{A}:=\left\{u \in W^{1, \infty}(\mathbb{R}): \operatorname{supp}(u)=[0, W], u^{\prime} \in\left\{\tan \left(-\theta_{m}+n \theta\right), n \in \mathcal{N}\right\} \text { a.e. in }(0, W)\right\},
$$

where the width $W$ depends on the function $u$, and only for simplicity of notation we will avoid writing $W(u)$. We denote by $\mathcal{F}(m)$ the infimum of the total energy at given mass $m$,

$$
\mathcal{F}(m):=\inf \{E(u): u \in \mathcal{A},|S(u)|=m\},
$$

where the mass (or volume) of the island is given by

$$
|S(u)|:=\int_{0}^{W} u(x) d x .
$$

Throughout the text, $C$ denotes a generic constant that may increase from line to line, depending only on $\theta_{m}, \theta$ and $\gamma_{0}=\gamma_{m}$, but not on $u$ or $W$. Moreover, for simplicity of notation, we set $S_{0} \frac{2}{\pi}=1=\gamma_{0}=\gamma_{m}$, even though all our results can be easily adapted to the case of realistic material parameters. Indeed, most of our result are of qualitative nature, and rely essentially on two charcateristics of the energy functional. First, due to the different scaling properties, at small volumes the surface energy dominates, while at large volumes the elastic energy wins. Second, we use the fact that the ratio $\gamma_{m} / \gamma_{0}$ is not too large, which ensures that the surface energy is always positive. Note that, in general, $\gamma_{m}$ is expected to be slightly different from $\gamma_{0}$ since microscopically, the interface between the substrate and the film consists of small steps (see [18] for a discussion). Summarizing, we will consider the following form for the elastic and the surface energies,

$$
E_{\mathrm{r}}(u):=\int_{0}^{W} \int_{0}^{W} u^{\prime}(x) u^{\prime}(y) \ln |x-y| d y d x, \quad E_{\mathrm{S}}(u):=\operatorname{length}(\operatorname{Graph}(u))-W .
$$

2.2. The "Killing Lemma". Most of our proofs of geometric properties of minimizers rely on the following "killing" strategy. The latter essentially quantifies the inequality

$$
\int_{a}^{b} f^{\prime}(x) g(x) d x \leq 0
$$

if $f \in W^{1, \infty}(a, b)$ has a maximum at $a$, and $g:(a, b) \rightarrow \mathbb{R}$ is positive and decreasing. Heuristically, if $f^{\prime}>0$ in some interval $(c, d) \subset(a, b)$, i.e., $f$ increases in $(c, d)$, then, since $f$ has a maximum at $a, f$ decreases by at least the same amount in $(a, c)$. By the monotonicity of $g$, the positive contributions to the integral are then "killed" by the preceding negative ones.

Lemma 2.1 (The Killing Lemma). Consider an arbitrary interval $(a, b) \subset \mathbb{R}, a<b$. Let $f$, $g:(a, b) \rightarrow \mathbb{R}$ be such that $f \in W^{1, \infty}(a, b)$ and $g$ is decreasing. 
(i) If $g \geq 0$ and $f(x) \leq f(a)$ for all $a \leq x \leq b$, then

$$
\int_{a}^{b} f^{\prime}(x) g(x) d x \leq 0 .
$$

(ii) If $g \geq 0$ and $0 \leq f(x) \leq \delta$ for all $a \leq x \leq b$, then

$$
\left|\int_{a}^{b} f^{\prime}(x) g(x) d x\right| \leq\left\|f^{\prime}\right\|_{L^{\infty}(a, b)} \int_{a}^{a+\frac{\delta}{\left\|f^{\prime}\right\|_{L^{\infty}}}} g(x) d x .
$$

In particular,

$$
\left|\int_{a}^{b} f^{\prime}(x) g(x) d x\right| \leq \delta g(a) .
$$

(iii) Suppose that $g$ is bounded below, or that $g \in L^{1}(a, b)$. If $f(x) \leq f(a)=f(b)$ for all $a \leq x \leq b$, then

$$
\int_{a}^{b} f^{\prime}(x) g(x) d x \leq 0 .
$$

If $f$ is not constant and $g$ is strictly decreasing, then the inequalities (2.2), (2.4) and (2.5) are strict.

Proof. We prove the three parts separately.

Step I. Proof of (i).

We define the sets

$$
\Sigma^{ \pm}:=\left\{x \in(a, b): f^{\prime}(x) \gtrless 0\right\},
$$

and the associated functions $\sigma^{ \pm}: \Sigma^{ \pm} \rightarrow \mathbb{R}^{+}$given by

$$
\sigma^{ \pm}(x):=\int_{\left\{z \in \Sigma^{ \pm} ; z<x\right\}}\left|f^{\prime}(z)\right| d z .
$$

If $\Sigma^{+}=\emptyset$ then $f^{\prime}(x) \leq 0$ for almost every $x \in(a, b)$, and thus

$$
\int_{a}^{b} \underbrace{f^{\prime}(x)}_{\leq 0} \underbrace{g(x)}_{\geq 0} d x \leq 0
$$

where the inequality is strict if $f$ is not constant and $g$ is strictly decreasing. Otherwise, note that $\sigma^{ \pm}$are increasing continuous functions. We set $x_{M}^{ \pm}:=\sup \left\{x: x \in \Sigma^{ \pm}\right\}$. Since $f(a) \geq f(b)$, we have $\sigma^{+}\left(x_{M}^{+}\right) \leq \sigma^{-}\left(x_{M}^{-}\right)$. We define $x^{*} \in \Sigma^{-}$by $\sigma^{-}\left(x^{*}\right)=\sigma^{+}\left(x_{M}^{+}\right)$, and set

$$
\Sigma_{*}^{-}:=\left\{x \in \Sigma^{-}: x \leq x^{*}\right\}
$$

Consider the map $\tau: \Sigma_{*}^{-} \rightarrow \Sigma^{+}$given by

$$
\sigma^{-}(x)=\sigma^{+}(\tau(x)) .
$$

Then $\tau$ is well-defined, bijective and strictly increasing. Furthermore, for almost every $x \in \Sigma_{*}^{-}$ we have $\tau(x)>x$, and

$$
\tau^{\prime}(x)=-\frac{f^{\prime}(x)}{f^{\prime}(\tau(x))} .
$$


We can then estimate

$$
\begin{aligned}
\int_{a}^{b} f^{\prime}(x) g(x) d x & =\int_{\Sigma_{*}^{-}} f^{\prime}(x) g(x) d x+\int_{\Sigma^{+}} f^{\prime}(x) g(x) d x+\int_{\Sigma^{-} \backslash \Sigma_{*}^{-}} \underbrace{f^{\prime}(x)}_{<0} \underbrace{g(x)}_{\geq 0} d x \\
& \leq \int_{\Sigma_{*}^{-}} f^{\prime}(x) g(x) d x+\int_{\Sigma^{+}} f^{\prime}(x) g(x) d x \\
& =\int_{\Sigma_{*}^{-}}\left(f^{\prime}(x) g(x)-f^{\prime}(\tau(x)) g(\tau(x)) \frac{f^{\prime}(x)}{f^{\prime}(\tau(x))}\right) d x \\
& =\int_{\Sigma_{*}^{-}} \underbrace{f^{\prime}(x)}_{<0} \underbrace{(g(x)-g(\tau(x)))}_{\geq 0} d x \leq 0,
\end{aligned}
$$

and also in this case we immediately derive the strict inequality if $f$ is not constant and $g$ is strictly decreasing.

Step II. Proof of (ii).

Let $\hat{x}$ be a global maximum point for $f$, i.e., $f(\hat{x}) \geq f(x)$ for all $x \in[a, b]$. By Step (i) we have

$$
\int_{\hat{x}}^{b} f^{\prime}(x) g(x) d x \leq 0
$$

and hence

$$
\int_{a}^{b} f^{\prime}(x) g(x) d x \leq \int_{a}^{\hat{x}} f^{\prime}(x) g(x) d x .
$$

In the interval $(a, \hat{x})$, consider the "mirror functions"

$$
\bar{f}(x):=f(-x+a+\hat{x}) \quad \text { and } \quad \bar{g}(x):=g(-x+a+\hat{x}) .
$$

Note that $\bar{f}(a) \geq \bar{f}(x)$ for all $x \in(a, \hat{x}), \bar{g}$ is positive and increasing, and

$$
\int_{a}^{\hat{x}} \bar{f}^{\prime}(x) \bar{g}(x) d x=-\int_{a}^{\hat{x}} f^{\prime}(x) g(x) d x .
$$

Defining now $\Sigma^{ \pm}, x^{*}$ and $\Sigma_{*}^{-}$as in Step I (with $\bar{f}$ and $(a, \hat{x})$ in place of $f$ and $(a, b)$ ), one has

$$
\int_{\Sigma^{-} \backslash \Sigma_{*}^{-}} \bar{f}^{\prime}(x) d x=\int_{a}^{\hat{x}} \bar{f}^{\prime}(x) d x \geq-\delta .
$$

Hence, since $\bar{g}$ is positive and increasing, and $\bar{f}$ is Lipschitz, we have

$$
\int_{\Sigma^{-} \backslash \Sigma_{*}^{-}} \bar{f}^{\prime}(x) \bar{g}(x) d x \geq-\left\|f^{\prime}\right\|_{L^{\infty}} \int_{\hat{x}-\frac{\delta}{\left\|f^{\prime}\right\|_{L^{\infty}}}}^{\hat{x}} \bar{g}(x) d x=-\left\|f^{\prime}\right\|_{L^{\infty}} \int_{a}^{a+\frac{\delta}{\left\|f^{\prime}\right\|_{L^{\infty}}}} g(x) d x .
$$

Putting together (2.6), (2.7) and (2.8) and changing variables as in Step I, we obtain that

$$
\begin{aligned}
\int_{a}^{b} f^{\prime}(x) g(x) d x & \leq-\int_{a}^{\hat{x}} \bar{f}^{\prime}(x) \bar{g}(x) d x \\
& =-\int_{\Sigma_{*}^{-}} \underbrace{\bar{f}^{\prime}(x)}_{<0} \underbrace{(\bar{g}(x)-\bar{g}(\tau(x)))}_{\leq 0} d x-\int_{\Sigma^{-} \backslash \Sigma_{*}^{-}} \bar{f}^{\prime}(x) \bar{g}(x) d x \\
& \leq-\int_{\Sigma^{-} \backslash \Sigma_{*}^{-}} \bar{f}^{\prime}(x) \bar{g}(x) d x \leq\left\|f^{\prime}\right\|_{L^{\infty}} \int_{a}^{a+\frac{\delta}{\left\|f^{\prime}\right\|_{L^{\infty}}}} g(x) d x
\end{aligned}
$$


Applying the above argument to $\tilde{g}:=g$ and $\tilde{f}:=\delta-f$, we obtain

$$
\int_{a}^{b} f^{\prime}(x) g(x) d x \geq-\left\|f^{\prime}\right\|_{L^{\infty}(a, b)} \int_{a}^{a+\frac{\delta}{\left\|f^{\prime}\right\|_{L} \infty}} g(x) d x,
$$

so the desired inequalities (2.3) and (2.4), the latter strict if $g$ is strictly decreasing, follow.

Step III. Proof of (iii).

Assume first that $g$ is bounded below. Since $f(a)=f(b)$, we have $\int_{a}^{b} f^{\prime}(x) d x=0$, and so we may replace $g$ by $g+\inf _{x \in(a, b)} g(x)$, which is positive and decreasing, and apply Step I.

Suppose now that $g$ is not bounded below, but belongs to $L^{1}$. For every $\varepsilon>0$, let us define the function $f_{\varepsilon}:(a, b) \rightarrow \mathbb{R}$ as

$$
f_{\varepsilon}(x)= \begin{cases}f(x) & \text { if } a<x<b-2 \varepsilon \\ f(2 x-b+2 \varepsilon) & \text { if } b-2 \varepsilon<x<b-\varepsilon, \\ f(b) & \text { if } b-\varepsilon<x<b .\end{cases}
$$

Since $f_{\varepsilon}$ is Lipschitz in $(a, b-\varepsilon), f_{\varepsilon}(a)=f_{\varepsilon}(b-\varepsilon)$, and $f_{\varepsilon}^{\prime}(x)=0$ in $(b-\varepsilon, b)$, while $g$ is decreasing and bounded below in $(a, b-\varepsilon)$ we have that

$$
\int_{a}^{b} f_{\varepsilon}^{\prime}(x) g(x) d x=\int_{a}^{b-\varepsilon} f_{\varepsilon}^{\prime}(x) g(x) d x \leq 0 .
$$

But by construction it now follows that

$$
\begin{aligned}
\left|\int_{a}^{b} f_{\varepsilon}^{\prime}(x) g(x) d x-\int_{a}^{b} f^{\prime}(x) g(x) d x\right| & =\left|\int_{b-2 \varepsilon}^{b-\varepsilon} f_{\varepsilon}^{\prime}(x) g(x) d x-\int_{b-2 \varepsilon}^{b} f^{\prime}(x) g(x) d x\right| \\
& \leq 3\left\|f^{\prime}\right\|_{L^{\infty}} \int_{b-2 \varepsilon}^{b}|g(x)| d x
\end{aligned}
$$

and the latter term is arbitrarily small for $\varepsilon \ll 1$ because $g \in L^{1}(a, b)$. This concludes the proof.

Remark 2.2. As an immediate consequence of the proof above, in the claim of Part (ii) the two terms $\left\|f^{\prime}\right\|_{L^{\infty}(a, b)}$ can be replaced by any larger constant. Indeed, in (2.8), $\left\|f^{\prime}\right\|_{L^{\infty}(a, b)}$ can be replaced by any larger constant, and the claim follows.

2.3. A bound on the length $W$. This section is devoted to proving an important geometrical property of the minimizers $u \in \mathcal{A}$ of the functional $E$, namely, that for every fixed volume $m$ the length $W$ of a low energy island is bounded above. This property has always been assumed in numerical simulations (see [18]), and it will also be useful here to ensure compactness of energy minimizing sequences. In fact, we will prove a stronger result, that is, the elastic energy of an island of mass $m$ and width $W$ explodes when $m$ is fixed and $W \rightarrow \infty$. Additionally, we can also show that, when the mass tends to 0 , then also the width $W$ of low energy islands tends to 0 . Recall that this behavior for small mass is a consequence of the non-zero miscut angle and is in contrast to the wetting effect (cf. [1]).

We start our analysis by considering an auxiliary special case. 
Lemma 2.3. For every $L>0$, there exists a constant $K=K(L)$ such that, for every $W>0$ and every L-Lipschitz function $u:[0, W] \rightarrow[0, \varepsilon]$ with $\varepsilon \leq \min , W / 8\}$, one has

$$
E_{r}(u)=\int_{0}^{W} \int_{0}^{W} u^{\prime}(y) u^{\prime}(x) \ln (|x-y|) d y d x \geq-K W\left(\varepsilon^{2}-\int_{0}^{\varepsilon} \ln (t) d t\right) .
$$

Proof. Since a function which is $L$-Lipschitz is also $\widetilde{L}$-Lipschitz for every $\widetilde{L}>L$, we can assume without loss of generality that $L \geq 1$. We subdivide the proof in several steps.

Step I. The estimate (2.10) for short intervals and the conclusion for $W \leq 1$.

We start by considering a "short" interval $I=(z, z+\ell) \subseteq(0, W)$, with $\ell \leq 1$, and we aim at estimating the integral

$$
\int_{I} \int_{I} u^{\prime}(y) u^{\prime}(x) \ln (|x-y|) d x d y
$$

Notice that, for every given $y \in I$, the function $x \mapsto-\ln (x-y)$ is positive and decreasing for $x \in(y, z+\ell)$. Thus, since $0 \leq u \leq \varepsilon$ and applying Lemma 2.1(ii), and also recalling Remark 2.2, we get

$$
\left|\int_{y}^{z+\ell} u^{\prime}(x) \ln (x-y) d x\right| \leq-L \int_{y}^{y+\frac{\varepsilon}{L}} \ln (x-y) d x=-L \int_{0}^{\frac{\varepsilon}{L}} \ln (t) d t \leq-L \int_{0}^{\varepsilon} \ln (t) d t .
$$

We deduce that

$$
\begin{aligned}
\left|\int_{z}^{z+\ell} \int_{y}^{z+\ell} u^{\prime}(y) u^{\prime}(x) \ln (x-y) d x d y\right| & \leq \int_{z}^{z+\ell}\left|u^{\prime}(y)\right|\left|\int_{y}^{z+\ell} u^{\prime}(x) \ln (x-y) d x\right| d y \\
& \leq-L^{2} \ell \int_{0}^{\varepsilon} \ln (t) d t .
\end{aligned}
$$

Assume now that $W \leq 1$. Applying (2.10) to the interval $I:=(0, W)$, we find

$$
\begin{aligned}
\int_{0}^{W} \int_{0}^{W} u^{\prime}(y) u^{\prime}(x) \ln (|x-y|) d y d x & =2 \int_{0}^{W} \int_{\{y: x<y<W\}} u^{\prime}(y) u^{\prime}(x) \ln (|x-y|) d y d x \\
& \geq 2 L^{2} W \int_{0}^{\varepsilon} \ln (t) d t \geq 2 L^{2} W \int_{0}^{\varepsilon} \ln (t) d t-2 L^{2} W \varepsilon^{2},
\end{aligned}
$$

and so (2.9) follows for $W \leq 1$ by choosing $K:=2 L^{2}$.

Step II. Proof of (2.14) in the case $W=2^{N+1} \geq 2, u(0)=u(W)=0, u(W / 2)=\varepsilon$.

We consider now the case in which $W \geq 2$ is a power of 2 , say $W=2^{N+1}$ with $N \geq 0$, and we also assume that $u(0)=u(W)=0$, and that $u(W / 2)=\varepsilon$. We aim at obtaining a lower bound for the mixed term

$$
\int_{0}^{2^{N}} \int_{2^{N}}^{2^{N+1}} u^{\prime}(x) u^{\prime}(y) \ln (x-y) d x d y
$$

Define $\phi:\left[0,2^{N}\right) \rightarrow \mathbb{R}$ by

$$
\phi(y):=\int_{2^{N}}^{2^{N+1}} u^{\prime}(x) \ln (|x-y|) d x=\int_{2^{N}}^{2^{N+1}} u^{\prime}(x) \ln (x-y) d x .
$$

By Lemma 2.1(i), and using the fact that $u(x) \leq \varepsilon=u(W / 2)=u\left(2^{N}\right), \phi$ is strictly increasing since

$$
\phi^{\prime}(y)=-\int_{2^{N}}^{2^{N+1}} u^{\prime}(x) \frac{1}{x-y} d x>0 \quad \text { for all } y \in\left(0,2^{N}\right) .
$$


We subdivide the interval $\left(0,2^{N}\right)$ into sets $A^{-}$and $A^{+},\left(0,2^{N}\right)=A^{-} \cup A^{+}$, with

$$
A^{+}:=\left\{y \in\left(0,2^{N}\right): \phi(y) \geq 0\right\} \text { and } A^{-}:=\left\{y \in\left(0,2^{N}\right): \phi(y)<0\right\} .
$$

Since $\phi$ is strictly increasing, we have $a^{-}<a^{+}$for all $a^{-} \in A^{-}$and $a^{+} \in A^{+}$, and each one of the sets could be empty (but not both, of course!). Let us first consider the interval $A^{-}$, assuming it is not empty. Observe that within $A^{-}$the points $y$ with positive derivative $u^{\prime}(y)$ are favorable for low elastic energy. Since $0=u(0) \leq u(x) \leq \varepsilon$ for all $x \in A^{-}$, and using the fact that $\phi$ is negative in $A^{-}$and increasing, we get by Lemma 2.1(ii)

$$
\int_{A^{-}} u^{\prime}(y) \phi(y) d y \geq \varepsilon \min _{y \in A^{-}} \phi(y)=\varepsilon \phi(0) .
$$

To estimate $\phi(0)=\int_{2^{N}}^{2^{N+1}} u^{\prime}(x) \ln (x) d x$, it suffices to note that $\ln (x)$ is positive and increasing in $\left(2^{N}, 2^{N+1}\right)$, so that by Lemma 2.1 (ii) we have $\phi(0) \geq-\varepsilon \ln \left(2^{N+1}\right)$. Inserting this estimate into (2.11), we obtain

$$
\int_{A^{-}} \int_{2^{N}}^{2^{N+1}} u^{\prime}(x) u^{\prime}(y) \ln (x-y) d x d y=\int_{A^{-}} u^{\prime}(y) \phi(y) d y \geq-\varepsilon^{2} \ln \left(2^{N+1}\right) .
$$

Note that this bound holds trivially also if $A^{-}=\emptyset$.

On $A^{+}$, the very opposite happens. In fact, since $\phi$ is positive and increasing on $A^{+}$, and since $u(x) \leq \varepsilon=u\left(2^{N}\right)$ for all $0 \leq x \leq 2^{N}$, by Lemma 2.1(i),

$$
\int_{A^{+}} u^{\prime}(y) \phi(y) d y \geq 0
$$

which holds trivially also if $A^{+}=\emptyset$. Putting together (2.12) and (2.13), we conclude that

$$
\int_{0}^{2^{N}} \int_{2^{N}}^{2^{N+1}} u^{\prime}(x) u^{\prime}(y) \ln (x-y) d x d y \geq-\varepsilon^{2} \ln \left(2^{N+1}\right) .
$$

Step III. Proof of (2.17) in the case $W=2^{N+1} \geq 2$.

We now remove the assumptions $u(0)=u(W)=0$ and $u(W / 2)=\varepsilon$, but we still assume that $W \geq 2$ is a power of 2 . We aim again at finding a bound on the mixed term $\int_{0}^{2^{N}} \int_{2^{N}}^{2^{N+1}} u^{\prime}(x) u^{\prime}(y) \ln (x-y) d x d y$. We will do so by modifying $u$ as sketched in Figure 3 . Precisely, we set $\tilde{u}:[0, W] \rightarrow[0, \varepsilon]$ as the function such that $\tilde{u}=u$ out of the four segments $\left[0, \varepsilon_{1}\right],\left[W / 2-\varepsilon_{2}, W / 2\right],\left[W / 2, W / 2+\varepsilon_{3}\right]$ and $\left[W-\varepsilon_{4}, W\right], \tilde{u}$ satisfies $\tilde{u}(0)=\tilde{u}(W)=0$ and $\tilde{u}(W / 2)=\varepsilon$,

$$
\tilde{u}^{\prime}-u^{\prime}:=\left\{\begin{aligned}
L & \text { in }\left(0, \varepsilon_{1}\right) \cup\left(W / 2-\varepsilon_{2}, W / 2\right), \\
-L & \text { in }\left(W / 2, W / 2+\varepsilon_{3}\right) \cup\left(W-\varepsilon_{4}, W\right),
\end{aligned}\right.
$$

and $\tilde{u}(x) \neq u(x)$ for all $x$ in the four segments. Note that these conditions uniquely determine $\varepsilon_{i}, i=1, \ldots, 4$, and thus $\tilde{u}$.

From the definition, using that $\max u \leq \varepsilon$ and $L \geq 1$, we have $0 \leq \varepsilon_{i} \leq \varepsilon$ for $1 \leq i \leq 4$, and actually $0 \leq \tilde{u} \leq \varepsilon$ on $[0, W]$. We can then apply Step II to $\tilde{u}$, which yields (see $(2.14)$ )

$$
\int_{0}^{2^{N}} \int_{2^{N}}^{2^{N+1}} \tilde{u}^{\prime}(x) \tilde{u}^{\prime}(y) \ln (x-y) d x d y \geq-\varepsilon^{2} \ln \left(2^{N+1}\right),
$$




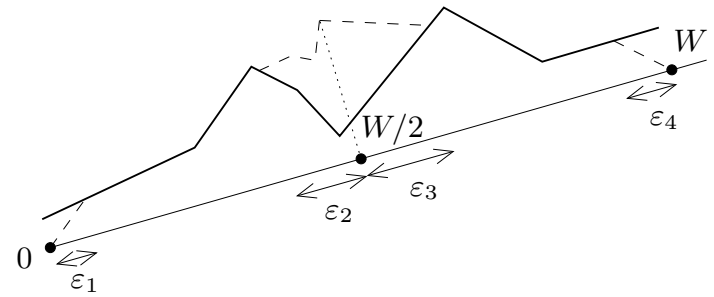

Figure 3. Modification $\tilde{u}$ of $u$ in Step III. Modifications are indicated by dashed lines.

and it remains to estimate the error incurred by our modification. Let us first check the term

$$
\int_{0}^{2^{N}} \int_{2^{N}}^{2^{N}+\varepsilon_{3}} u^{\prime}(y)\left(u^{\prime}(x)-\tilde{u}^{\prime}(x)\right) \ln (x-y) d x d y=L \int_{0}^{2^{N}} u^{\prime}(y) \tau(y) d y,
$$

having set for brevity

$$
\tau(y):=\int_{2^{N}}^{2^{N}+\varepsilon_{3}} \ln (x-y) d x \quad \text { for } \quad y \in\left(0,2^{N}\right) .
$$

As above, we define the sets $A^{+}:=\{\tau \geq 0\}$ and $A^{-}:=\{\tau<0\}$. Then, by Lemma 2.1(ii), and using the fact that $\tau$ is decreasing, we have

$$
\int_{A^{+}} u^{\prime}(y) \tau(y) d y \geq-\varepsilon \tau(0)=-\varepsilon\left(-\varepsilon_{3}+2^{N} \ln \left(1+\frac{\varepsilon_{3}}{2^{N}}\right)+\varepsilon_{3} \ln \left(2^{N}+\varepsilon_{3}\right)\right) \geq-\varepsilon^{2} \ln \left(2^{N}+\varepsilon\right),
$$

while

$$
\int_{A^{-}} u^{\prime}(y) \tau(y) d y \geq \varepsilon \tau\left(2^{N}\right) \geq \varepsilon \int_{0}^{\varepsilon} \ln (t) d t,
$$

and in turn this implies by (2.16) that

$$
\int_{0}^{2^{N}} \int_{2^{N}}^{2^{N}+\varepsilon_{3}} u^{\prime}(y)\left(u^{\prime}(x)-\tilde{u}^{\prime}(x)\right) \ln (x-y) d x d y \geq-L \varepsilon^{2} \ln \left(2^{N}+\varepsilon\right)+L \varepsilon \int_{0}^{\varepsilon} \ln (t) d t .
$$

Arguing in the very same way in the other intervals where $u$ has been modified, we obtain

$$
\int_{0}^{2^{N}} \int_{2^{N}}^{2^{N+1}}\left(u^{\prime}(y) u^{\prime}(x)-\tilde{u}^{\prime}(y) \tilde{u}^{\prime}(x)\right) \ln (x-y) d x d y \geq-4 L \varepsilon^{2} \ln \left(2^{N}+\varepsilon\right)+4 L \varepsilon \int_{0}^{\varepsilon} \ln (t) d t,
$$

which, combined with (2.15), yields the estimate

$$
\int_{0}^{2^{N}} \int_{2^{N}}^{2^{N+1}} u^{\prime}(y) u^{\prime}(x) \ln (x-y) d x d y \geq-\varepsilon^{2}(4 L+1) \ln \left(2^{N+1}\right)+4 L \varepsilon \int_{0}^{\varepsilon} \ln (t) d t .
$$

Step IV. Conclusion for $W=2^{N+1} \geq 2$.

We are now ready to conclude the proof for the case in which $W=2^{N+1} \geq 2$ is a power of 2 . The inequality (2.17) from Step III allows to estimate the "mixed part" of the integral in (2.9), namely, the one concerning $0 \leq y \leq 2^{N}$ and $2^{N} \leq x \leq 2^{N+1}$. We now decompose the set $\left\{(x, y): 0 \leq y \leq x \leq 2^{N+1}\right\}$ in the following way (see Figure 4 for a visualization and recall 
that $\left.W=2^{N+1}\right)$ :

$$
\begin{aligned}
& \{(x, y): 0 \leq y \leq x \leq W\}=\left[\left(\frac{1}{2} W, W\right) \times\left(0, \frac{1}{2} W\right)\right] \cup \\
\cup & {\left[\left(\frac{1}{4} W, \frac{1}{2} W\right) \times\left(0, \frac{1}{4} W\right)\right] \cup\left[\left(\frac{3}{4} W, W\right) \times\left(\frac{1}{2} W, \frac{3}{4} W\right)\right] \cup } \\
\cup & \cdots \cup \\
\cup & {\left[\left(\frac{1}{2^{N+1}} W, \frac{1}{2^{N}} W\right) \times\left(0, \frac{1}{2^{N+1}} W\right)\right] \cup \cdots \cup\left[\left(\frac{2^{N+1}-1}{2^{N+1}} W, W\right) \times\left(\frac{2^{N}}{2^{N+1}} W, \frac{2^{N+1}-1}{2^{N+1}} W\right)\right] }
\end{aligned}
$$

where $R$ consists of $2^{N+1}$ isosceles right-angled triangles of area $1 / 2$ each. We now apply $(2.17)$ iteratively. First consider the restriction of $u$ to the interval $\left(0,2^{N}\right)$ to obtain

$$
\int_{0}^{2^{N-1}} \int_{2^{N-1}}^{2^{N}} u^{\prime}(y) u^{\prime}(x) \ln (x-y) d x d y \geq-\varepsilon^{2}(4 L+1) \ln \left(2^{N}\right)+4 L \varepsilon \int_{0}^{\varepsilon} \ln (t) d t,
$$

and the same can be done in the interval $\left(2^{N}, 2^{N+1}\right)$. Therefore, in this "second iteration step" we obtain twice the contribution from (2.17) with $2^{N+1}$ replaced by $2^{N}$ and, similarly, in the "third iteration step" we get four times the contribution with $2^{N+1}$ replaced by $2^{N-1}$, and so on (see Figure 4 to visualize the consequent sets of pairs $(x, y) \in[0, W] \times[0, W]$ that one can reach with these iterations). After $N+1$ iterations, we recover the whole set $\left\{0 \leq y \leq x \leq 2^{N+1}\right\}$ except the $2^{N+1}$ small triangles below the diagonal. Adding up, one has

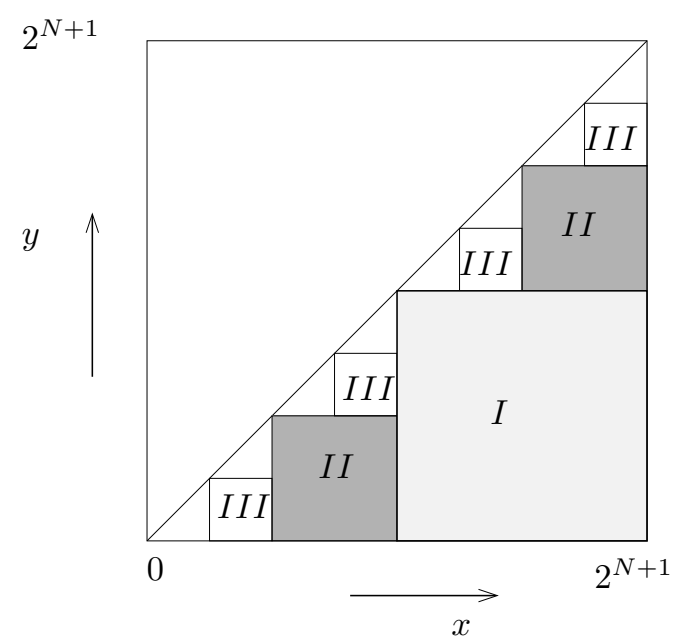

FIgURE 4. Domains of integration in Step IV

$$
\begin{aligned}
& \frac{1}{2} \int_{0}^{2^{N+1}} \quad \int_{0}^{2^{N+1}} u^{\prime}(x) u^{\prime}(y) \ln (|x-y|) d x d y=\iint_{y<x} u^{\prime}(x) u^{\prime}(y) \ln (x-y) d x d y \\
& \geq-\varepsilon^{2}(4 L+1)\left(\ln \left(2^{N+1}\right)+2 \ln \left(2^{N}\right)+4 \ln \left(2^{N-1}\right)+\cdots+2^{N} \ln (2)\right) \\
& \quad+4 L\left(2^{N+1}-1\right) \varepsilon \int_{0}^{\varepsilon} \ln (t) d t+\sum_{j=0}^{2^{N+1}-1} \int_{j}^{j+1} \int_{y}^{j+1} u^{\prime}(x) u^{\prime}(y) \ln (x-y) d x d y .
\end{aligned}
$$


Concerning the first term, we rewrite the sum as

$$
\begin{aligned}
\ln \left(2^{N+1}\right)+2 \ln \left(2^{N}\right)+\cdots+2^{N} \ln (2) & =2^{N+1} \ln (2)\left(\frac{1}{2}+\frac{2}{4}+\frac{3}{8}+\cdots+\frac{N+1}{2^{N+1}}\right) \\
& \leq 2^{N+2} \ln (2) .
\end{aligned}
$$

Concerning the second term of (2.18), we observe that

$$
\left(2^{N+1}-1\right) \varepsilon \int_{0}^{\varepsilon} \ln (t) d t \geq \frac{W}{2} \varepsilon \int_{0}^{\varepsilon} \ln (t) d t .
$$

Then, for each of the $2^{N+1}$ integrals in the last term we can apply the estimate (2.10) from Step I with $z=j$ and $\ell=1$ to obtain

$$
\int_{j}^{j+1} \int_{y}^{j+1} u^{\prime}(y) u^{\prime}(x) \ln (x-y) d x d y \geq L^{2} \int_{0}^{\varepsilon} \ln (t) d t .
$$

Finally, inserting (2.19), (2.20) and (2.21) into (2.18), we conclude that

$$
\begin{aligned}
\int_{0}^{W} \int_{0}^{W} u^{\prime}(x) & u^{\prime}(y) \ln (|x-y|) d x d y \\
& \geq-2^{N+3} \varepsilon^{2}(4 L+1) \ln (2)+4 L W \varepsilon \int_{0}^{\varepsilon} \ln (t) d t+2^{N+2} L^{2} \int_{0}^{\varepsilon} \ln (t) d t \\
& \geq K(L) W\left(-\varepsilon^{2}+\int_{0}^{\varepsilon} \ln (t) d t\right),
\end{aligned}
$$

and we have reached (2.9).

Step $V$. Conclusion for $W>1$.

We are now left to consider the case of a generic $W>1$. It suffices to define $\widetilde{W}:=2^{N+1}$ as the smallest power of 2 greater than $W$, i.e., $2^{N} \leq W<2^{N+1}=\widetilde{W}$. Extend $u$ as $\tilde{u}:(0, \widetilde{W}) \rightarrow[0, \varepsilon]$ by setting $\tilde{u}(x):=u(\min \{x, W\})$. Applying the result of Step IV to $\tilde{u}$, we have

$$
\begin{aligned}
\int_{0}^{W} \int_{0}^{W} u^{\prime}(x) u^{\prime}(y) & \ln (|x-y|) d x d y=\int_{0}^{\widetilde{W}} \int_{0}^{\widetilde{W}} \tilde{u}^{\prime}(x) \tilde{u}^{\prime}(y) \ln (|x-y|) d x d y \\
& \geq K(L) \widetilde{W}\left(-\varepsilon^{2}+\int_{0}^{\varepsilon} \ln (t) d t\right) \geq 2 K(L) W\left(-\varepsilon^{2}+\int_{0}^{\varepsilon} \ln (t) d t\right),
\end{aligned}
$$

and this concludes the proof.

We now consider the whole energy, seeking again a lower bound.

Proposition 2.4. Let $m>0$. There exists $W_{0}=W_{0}(m)>0$, depending continuously on $m$, such that $E(u) \geq \kappa W$ for all classically admissible functions $u$ with $|S(u)|=m$ and $W \geq W_{0}$, where $\kappa>0$ is a purely geometric constant, depending only on $\theta$ and $\theta_{m}$.

Proof. Let $u \in \mathcal{A}$ with $|S(u)|=m$. We consider the elastic and the surface energies as given in (2.1) separately. By the constraint on the slopes, we have $\left|u^{\prime}(x)\right| \geq \tilde{\kappa}>0$ with some $\tilde{\kappa}$ that depends only on $\theta$ and $\theta_{m}$. Hence there is a geometrical constant $\kappa=\kappa\left(\theta, \theta_{m}\right)>0$ such that

$$
E_{\mathrm{S}}(u) \geq 2 \kappa W .
$$


On the other hand, functions in $\mathcal{A}$ are uniformly $L$-Lipschitz with a constant $L=L\left(\theta, \theta_{m}, \mathcal{N}\right)$, and so by Lemma 2.3 one has (by choosing $\kappa>0$ sufficiently small)

$$
E_{\mathrm{r}}(u) \geq-\frac{\kappa}{4} W \quad \text { if }\|u\|_{L^{\infty}} \leq \min \left\{1, \frac{W}{8}\right\} .
$$

Hence, the assertion follows immediately if $\|u\|_{L^{\infty}}$ is small enough, irrespectively of $m$. Let now $\bar{\varepsilon} \leq 1$ be a small positive number, depending on $m$, that will be specified at the end of the proof. Since $u$ is $L$-Lipschitz, it is possible to find a constant $\varepsilon$ depending on $u$, smaller than $\bar{\varepsilon}$ but arbitrarily close to it, such that $[0, W]$ is the essential union of finitely many open intervals $B_{0}$, $A_{1}, B_{1}, A_{2}, \ldots, A_{N}, B_{N}$ (see Figure 5 ), where the right extreme point of one interval coincides with the left extreme point of the consecutive one, $u>\varepsilon$ on every $A_{i}$, and $u<\varepsilon$ on every $B_{i}$ (note that this is true for all real numbers $\varepsilon$ except a negligible set). Set $A:=\cup_{i} A_{i}$ and $B:=\cup_{i} B_{i}$. Note that if $\|u\|_{L^{\infty}}<\bar{\varepsilon}$, then $A=\emptyset$. We have

$$
\begin{aligned}
E_{\mathrm{r}}(u)=\int_{B} \int_{B} u^{\prime}(x) u^{\prime}(y) \ln (|x-y|) d y d x+\int_{A} & \int_{A} u^{\prime}(x) u^{\prime}(y) \ln (|x-y|) d y d x \\
& +2 \int_{A} \int_{B} u^{\prime}(x) u^{\prime}(y) \ln (|x-y|) d y d x,
\end{aligned}
$$

and we will consider the three terms separately.

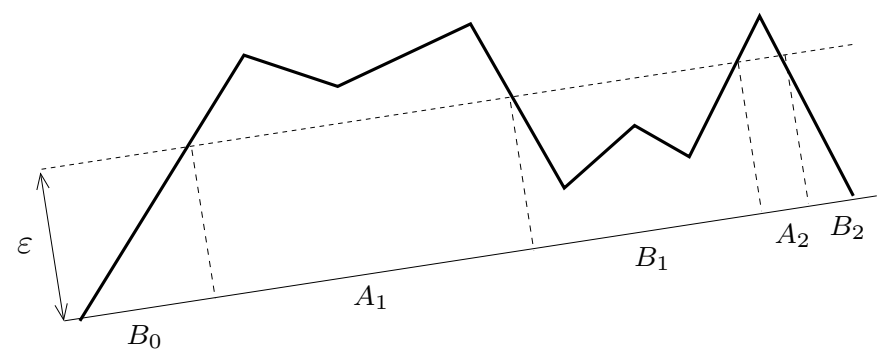

FiguRE 5. Definition of the intervals $A_{i}$ and $B_{i}$.

Step I. $x, y \in B$.

Set $\bar{u}:=u \wedge \varepsilon=\min \{u, \varepsilon\}$, so that by (2.23) we have

$$
\int_{B} \int_{B} u^{\prime}(x) u^{\prime}(y) \ln (|x-y|) d x d y=\int_{0}^{W} \int_{0}^{W} \bar{u}^{\prime}(x) \bar{u}^{\prime}(y) \ln (|x-y|) d x d y \geq-\frac{\kappa}{4} W,
$$

if $W \geq 8 \varepsilon$.

Step II. $x, y \in A$.

For any interval $A_{i}$,

$$
\begin{aligned}
\left|\int_{A_{i}} \int_{A_{i}} u^{\prime}(x) u^{\prime}(y) \ln (|x-y|) d x d y\right| & \leq 2\left\|u^{\prime}\right\|_{L^{\infty}}^{2} \int_{0}^{\left|A_{i}\right|} \int_{0}^{\left|A_{i}\right|}|\ln (t)| d t d y \\
& \leq 2\left\|u^{\prime}\right\|_{L^{\infty}}^{2}\left|A_{i}\right| \int_{0}^{\left|A_{i}\right|}|\ln (t)| d t .
\end{aligned}
$$


By the volume constraint $|S(u)|=m$ we have $\sum_{i}\left|A_{i}\right| \leq \frac{m}{\varepsilon}$, and by the constraint on the slopes, we have $\left\|u^{\prime}\right\|_{L^{\infty}} \leq c$. If $W \geq 8 c^{2} \frac{m}{\kappa \varepsilon} \int_{0}^{\frac{m}{\varepsilon}}|\ln (t)| d t$, then

$$
\begin{aligned}
\sum_{i}\left|\int_{A_{i}} \int_{A_{i}} u^{\prime}(x) u^{\prime}(y) \ln (|x-y|) d x d y\right| & \leq 2\left\|u^{\prime}\right\|_{L^{\infty}}^{2} \sum_{i}\left|A_{i}\right| \int_{0}^{\left|A_{i}\right|}|\ln (t)| d t \\
& \leq 2 c^{2} \frac{m}{\varepsilon} \int_{0}^{\frac{m}{\varepsilon}}|\ln (t)| d t \leq \frac{\kappa}{4} W .
\end{aligned}
$$

Note that the lower bound on $W$, i.e., $W \geq 8 c^{2} \frac{m}{\kappa \varepsilon} \int_{0}^{\frac{m}{\varepsilon}}|\ln (t)| d t$, depends continuously on $m$. Moreover,

$$
\int_{A_{1}} \int_{A \backslash A_{1}} u^{\prime}(x) u^{\prime}(y) \ln (|x-y|) d y d x=\int_{A \backslash A_{1}} u^{\prime}(y) \phi(y) d y,
$$

where $\phi(y):=\int_{A_{1}} u^{\prime}(x) \ln (y-x) d x$. By Lemma 2.1(iii), $\phi>0$ and $\phi^{\prime}<0$, and thus by Lemma 2.1(i),

$$
\int_{A_{1}} \int_{A \backslash A_{1}} u^{\prime}(x) u^{\prime}(y) \ln (|x-y|) d x d y>0
$$

Recursively,

$$
\sum_{i} \int_{A_{i}} \int_{A \backslash A_{i}} u^{\prime}(x) u^{\prime}(y) \ln (|x-y|) d x d y>0 .
$$

Hence, by (2.25) and (2.26),

$$
\int_{A} \int_{A} u^{\prime}(x) u^{\prime}(y) \ln (|x-y|) d x d y \geq \sum_{i} \int_{A_{i}} \int_{A_{i}} u^{\prime}(x) u^{\prime}(y) \ln (|x-y|) d x d y \geq-\frac{\kappa}{4} W .
$$

Step III. $x \in A$ and $y \in B$.

Consider an index $0 \leq i \leq N$, and write

$$
\int_{B_{i}} \int_{\bigcup_{j>i} A_{j}} u^{\prime}(x) u^{\prime}(y) \ln (|x-y|) d x d y=\int_{B_{i}} u^{\prime}(y) \phi(y) d y
$$

with $\phi(y):=\int_{\bigcup_{j>i} A_{j}} u^{\prime}(x) \ln (x-y) d x$. By Lemma 2.1(iii), $\phi<0$ and $\phi^{\prime}<0$. Let $P$ be the last point in $B_{i}$. Since $u \leq \varepsilon$ in $B_{i}$, by Lemma 2.1(ii)

$$
\int_{B_{i}} \int_{\bigcup_{j>i} A_{j}} u^{\prime}(x) u^{\prime}(y) \ln (|x-y|) d x d y \geq \varepsilon \phi(P) .
$$

Set $\tilde{u}:=u \vee \varepsilon=\max \{u, \varepsilon\}$. Then

$$
\phi(P)=\int_{[P, W] \cap\left(\cup A_{i}\right)} u^{\prime}(x) \ln (x-P) d x=\int_{P}^{W} \tilde{u}^{\prime}(x) \ln (x-P) d x,
$$

and since $\|\tilde{u}\|_{L^{\infty}} \leq \sqrt{L m}$ by the constraint on the slopes, by Lemma 2.1(ii) (applied with $\delta=\sqrt{L m}$, we get

$$
\begin{aligned}
|\phi(P)| & =\left|\int_{P}^{W} \tilde{u}^{\prime}(x) \ln (x-P) d x\right| \\
& \leq \int_{P}^{P+1}\left|\tilde{u}^{\prime}(x)\right||\ln (x-P)| d x+\left|\int_{P+1}^{W} \tilde{u}^{\prime}(x) \ln (x-P) d x\right| \leq C(1+\sqrt{m} \ln (W)),
\end{aligned}
$$


where $C$ depends only on $\left\|u^{\prime}\right\|_{L^{\infty}}$, i.e., on $\theta, \theta_{m}$ and $\mathcal{N}$. Inserting this estimate into (2.28) yields

$$
\int_{B_{i}} \int_{\cup_{j>i} A_{j}} u^{\prime}(x) u^{\prime}(y) \ln (|x-y|) d x d y \geq-C(1+\sqrt{m} \ln (W)) \varepsilon .
$$

By symmetry, we obtain the analogue estimate for $\bigcup_{j \leq i} A_{j}$. Thus,

$$
\int_{B_{i}} \int_{A} u^{\prime}(x) u^{\prime}(y) \ln (|x-y|) d x d y \geq-C(1+\sqrt{m} \ln (W)) \varepsilon,
$$

where again $C$ is a purely geometric constant. Recall that we can assume $A \neq \emptyset$ since otherwise, as we already observed, the thesis follows by $(2.22)$ and (2.23). Hence, every $B_{i}$ has at least one extreme point where $u$ takes the value $\varepsilon$. If $\left|B_{i}\right| \geq \frac{\varepsilon}{L}$, then by the constraint on the slopes, $\int_{B_{i}} u(x) d x \geq \frac{\varepsilon^{2}}{2 L}$, so that there are at most $\frac{2 m L}{\varepsilon^{2}}$ such $B_{i}$ 's. Thus by (2.29),

$$
\int_{\bigcup_{\left|B_{i}\right| \geq \frac{\varepsilon}{L}} B_{i}} \int_{A} u^{\prime}(x) u^{\prime}(y) \ln (|x-y|) d x d y \geq-C(1+\sqrt{m} \ln (W)) \frac{m}{\varepsilon} \geq-\frac{\kappa}{8} W
$$

provided $W \geq \widetilde{W}$, where $\widetilde{W}$ depends continuously on $m$. If $\left|B_{i}\right|<\frac{\varepsilon}{L}$, then $u=\varepsilon$ at both ends of $B_{i}$. Set $\delta:=\left|B_{i}\right|$ and consider

$$
\int_{B_{i}} \int_{\bigcup_{j>i} A_{j}} u^{\prime}(y) u^{\prime}(x) \ln (y-x) d y d x=\int_{\bigcup_{j>i} A_{j}} u^{\prime}(y) \psi(y) d y
$$

with $\psi(y):=\int_{B_{i}} u^{\prime}(x) \ln (y-x) d x$. Let $\hat{u}: B_{i} \rightarrow \mathbb{R}$ be defined as

$$
\hat{u}(x):= \begin{cases}\varepsilon-L(x-P+\delta) & \text { if } P-\delta \leq x \leq P-\frac{\delta}{2}, \\ \varepsilon+L(x-P) & \text { if } P-\frac{\delta}{2} \leq x \leq P\end{cases}
$$

and notice that $\hat{u} \leq u$ in $[P-\delta, P]$ but $\hat{u}(x)=u(x)$ both at $x=P-\delta$ and $x=P$. Let $\hat{\psi}$ be the same function as $\psi$, with $\hat{u}$ in place of $u$. Applying Lemma 2.1(iii), for every $y \in \bigcup_{j>i} A_{j}$ we obtain that $\hat{\psi}(y) \leq 0, \hat{\psi}^{\prime}(y) \geq 0$, and

$$
\begin{aligned}
& (\psi-\hat{\psi})(y)=\int_{B_{i}}\left(u^{\prime}(x)-\hat{u}^{\prime}(x)\right) \ln (y-x) d x \geq 0, \\
& (\psi-\hat{\psi})^{\prime}(y)=\int_{B_{i}} \frac{u^{\prime}(x)-\hat{u}^{\prime}(x)}{y-x} d x \leq 0 .
\end{aligned}
$$

As a consequence, since the value of $\tilde{u}:=u \vee \varepsilon$ is $\varepsilon$ both at $P$ and $W$, again Lemma 2.1 applied twice yields

$$
\int_{\bigcup_{j>i} A_{j}} u^{\prime}(y) \psi(y) d y=\int_{P}^{W} \tilde{u}^{\prime}(y) \psi(y) d y \geq \int_{P}^{W} \tilde{u}^{\prime}(y) \hat{\psi}(y) d y \geq L \int_{P}^{P+\sqrt{\frac{m}{L}}} \hat{\psi}(y) d y,
$$

using again that $\|u\|_{L^{\infty}} \leq \sqrt{L m}$. Since

$$
\hat{\psi}(y)=L\left(\int_{P-\delta}^{P-\delta / 2}-\ln (y-x) d x+\int_{P-\delta / 2}^{P} \ln (y-x) d x\right),
$$


we have

$$
\begin{aligned}
\frac{1}{L} \int_{P}^{P+\sqrt{\frac{m}{L}}} \hat{\psi}(y) d y & =\int_{P}^{P+\sqrt{\frac{m}{L}}}\left(\int_{P-\delta}^{P-\delta / 2}-\ln (y-x) d x+\int_{P-\delta / 2}^{P} \ln (y-x) d x\right) d y \\
& =-\int_{0}^{\sqrt{\frac{m}{L}}}\left(\int_{\delta / 2}^{\delta} \ln (y+x) d x-\int_{0}^{\delta / 2} \ln (y+x) d x\right) d y \\
& =-\int_{0}^{\sqrt{\frac{m}{L}}} \int_{0}^{\delta / 2} \ln \left(1+\frac{\delta}{2(y+x)}\right) d x d y \\
& \geq-\frac{\delta}{2} \int_{0}^{\sqrt{\frac{m}{L}}} \int_{0}^{\delta / 2} \frac{1}{y+x} d x d y=-\frac{\delta}{2} \int_{0}^{\sqrt{\frac{m}{L}}} \ln \left(1+\frac{\delta}{2 y}\right) d y \\
& =-\frac{\delta \sqrt{m}}{2 \sqrt{L}} \ln \left(1+\frac{\delta}{2 \sqrt{m / L}}\right)-\frac{\delta^{2}}{4} \ln \left(1+\frac{2 \sqrt{m}}{\delta \sqrt{L}}\right) \geq-\frac{\kappa}{8 L^{2}} \delta,
\end{aligned}
$$

where the last inequality holds if $\delta=\left|B_{i}\right|$ is small enough. Since, by construction, $\delta<\frac{\varepsilon}{L}$, the validity of the estimate above is true provided that the constant $\bar{\varepsilon}$ is chosen small enough. Putting together (2.31), (2.32) and (2.33), we find that

$$
\int_{B_{i}} \int_{\bigcup_{j>i} A_{j}} u^{\prime}(y) u^{\prime}(x) \ln (y-x) d y d x \geq-\frac{\kappa}{16} \delta=-\frac{\kappa}{16}\left|B_{i}\right|
$$

By symmetry, we deduce that

$$
\int_{B_{i}} \int_{A} u^{\prime}(y) u^{\prime}(x) \ln (|y-x|) d y d x \geq-\frac{\kappa}{8}\left|B_{i}\right|,
$$

and, finally, adding over the "short" intervals $B_{i}$, we conclude that

$$
\int_{\bigcup_{\left|B_{i}\right|<\frac{\varepsilon}{L}} B_{i}} \int_{A} u^{\prime}(x) u^{\prime}(y) \ln (|x-y|) d x d y \geq-\frac{\kappa}{8} W .
$$

Step IV. Conclusion.

Combining (2.24), (2.27), (2.30) and (2.34), we have that $E_{\mathrm{r}}(u) \geq-\kappa W$ if $W \geq W_{0}$, where $W_{0}$ depends continuously on $m$, and hence by $(2.22)$ we conclude that $E(u)=E_{\mathrm{S}}(u)+E_{\mathrm{r}}(u) \geq$ $\kappa W$.

We deduce that low-energy profiles are uniformly compactly supported. For that, we need the estimate $\underline{\lim }_{m \rightarrow 0} \mathcal{F}(m) \leq 0$, which will be improved to $\lim _{m \rightarrow 0} \mathcal{F}(m)=0$ in Corollary 2.7, and finally, we will show that the unique minimizer for small volume is given by the half-pyramid (see Section 7).

Lemma 2.5. We have

$$
\varliminf_{m \rightarrow 0} \mathcal{F}(m) \leq 0
$$

Proof. It suffices to construct admissible functions $u_{m}$ with $\left|S\left(u_{m}\right)\right|=m$ and $E\left(u_{m}\right) \leq C \sqrt{m}$ with some $C>0$ independent of $m$. Let $\lambda_{1}:=\tan \left(-\theta_{m}+\theta\right)>0$ and $\lambda_{2}:=\tan \left(-\theta_{m}\right)<0$ be 
the minimal positive and the maximal negative admissible slopes, respectively, and consider the half-pyramid

$$
u_{m}(x):=\left\{\begin{array}{ll}
\lambda_{1} x, & \text { if } 0 \leq x \leq \sqrt{\frac{2 m \lambda_{2}}{\lambda_{1} \lambda_{2}-\lambda_{1}^{2}}} \\
\lambda_{2} x+\sqrt{\frac{2 m \lambda_{2}\left(\lambda_{2}-\lambda_{1}\right)}{\lambda_{1}}}, & \text { if } \sqrt{\frac{2 m \lambda_{2}}{\lambda_{1} \lambda_{2}-\lambda_{1}^{2}}} \leq x \leq \sqrt{\frac{2 m\left(\lambda_{2}-\lambda_{1}\right)}{\lambda_{1} \lambda_{2}}}
\end{array} .\right.
$$

Then there are constants $C>0$ independent of $m$ such that $\left|u_{m}^{\prime}\right| \leq C$ almost everywhere, and $W_{m}=\left|\operatorname{supp}\left(u_{m}\right)\right| \leq C \sqrt{m}$. Therefore,

$$
E_{\mathrm{S}}\left(u_{m}\right) \leq C \sqrt{m} \rightarrow 0 \quad \text { as } m \rightarrow 0 .
$$

On the other hand, to estimate the elastic energy, we use that for any $a<b<c<d$, we have

$$
\begin{aligned}
& \int_{a}^{b} \int_{c}^{d} \ln |x-y| d x d y=-\frac{3}{2}(b-a)(d-c)+ \\
+ & \frac{1}{2}\left((c-b)^{2} \ln (c-b)+(d-a)^{2} \ln (d-a)-(d-b)^{2} \ln (d-b)-(c-a)^{2} \ln (c-a)\right),
\end{aligned}
$$

which implies that there is a constant $C$, which depends only on $\lambda_{1}$ and $\lambda_{2}$, such that for small $m>0$,

$$
E_{\mathrm{r}}\left(u_{m}\right) \leq C m|\ln m| \rightarrow 0 .
$$

Hence $E\left(u_{m}\right) \rightarrow 0$ as $m \rightarrow 0$, which concludes the proof.

Corollary 2.6. For any $m, r>0$ there exists $\bar{W}=\bar{W}(m, r)$, depending continuously on $m$ and $r$, such that if an admissible function $u \in \mathcal{A}$ with mass $m$ has low energy, precisely $E(u) \leq \mathcal{F}(m)+r$, then $W \leq \bar{W}$. Moreover, if $m \rightarrow 0$ and $r \rightarrow 0$, then $\bar{W}(m, r) \rightarrow 0$.

Proof. The first part of the statement is an immediate consequence of Proposition 2.4. For the second part take $m, r \ll 1$ and let $u$ be a classically admissible function with mass $m$ and energy satisfying $E(u) \leq \mathcal{F}(m)+r$. In particular, since $\varliminf_{m \rightarrow 0} \mathcal{F}(m) \leq 0$ by Lemma 2.5, for every $\varepsilon>0$ there is $m_{0}>0$ such that $E(u) \leq \varepsilon$ if $m<m_{0}$. Further, suppose $u(\bar{x})=\|u\|_{L^{\infty}}$, and let $\Lambda:=\tan \left(-\theta_{m}+n_{+} \theta\right)$ be the largest admissible slope. Then $u(x) \geq \Lambda x+\left(\|u\|_{L^{\infty}}-\Lambda \bar{x}\right)$ if $\frac{1}{\Lambda}\left(\Lambda \bar{x}-\|u\|_{L^{\infty}}\right) \leq x \leq \bar{x}$, and thus the volume is bounded below by the volume of a triangle with base $\|u\|_{L^{\infty}} / \Lambda$ and height $\|u\|_{L^{\infty}}$, i.e.,

$$
m \geq \int_{\bar{x}-\|u\|_{L \infty} / \Lambda}^{\bar{x}} u(y) d y \geq \frac{1}{2}\|u\|_{L^{\infty}}^{2} / \Lambda .
$$

We hence consider separately two cases. First, if $\|u\|_{L^{\infty}} \leq W / 8$, then with the notation of the proof of Proposition 2.4 we have $E_{\mathrm{S}}(u) \geq 2 \kappa W$ (see (2.22)) and $E_{\mathrm{r}}(u) \geq-\frac{\kappa}{4} W$ (see (2.23)), and thus $E(u) \geq \frac{7}{4} \kappa W$. Hence, $W$ is surely as small as desired (provided $m$ and $r$ are small enough). Second, if $\|u\|_{L^{\infty}}>W / 8$, then in particular by (2.36) we have

$$
W<8\|u\|_{L^{\infty}} \leq 8 \sqrt{2 \Lambda m},
$$

which is again arbitrarily small if $m \ll 1$.

Corollary 2.7. $\lim _{m \rightarrow 0} \mathcal{F}(m)=0$. 
Proof. Let $u_{m}$ be such that $E\left(u_{m}\right) \leq \mathcal{F}(m)+m$. Then by Corollary 2.6, for $m$ small, we have $W_{m} \ll 1$, and thus also (with the notation of the proof of Corollary 2.6)

$$
\left\|u_{m}\right\|_{L^{\infty}}=u_{m}(\bar{x})=\int_{0}^{\bar{x}} u_{m}^{\prime}(y) d y \leq W_{m} \Lambda \ll 1 .
$$

Further, we have by the slope constraint,

$$
E_{\mathrm{S}}\left(u_{m}\right)=\int_{0}^{W_{m}}\left(\sqrt{1+\left|u_{m}^{\prime}(y)\right|^{2}}-1\right) d y \leq C W_{m} \rightarrow 0 \quad \text { as } \quad W_{m} \rightarrow 0,
$$

and also $E_{\mathrm{r}}\left(u_{m}\right) \rightarrow 0$ as $W_{m} \rightarrow 0$ since

$$
\int_{0}^{W_{m}} \int_{0}^{W_{m}} u_{m}^{\prime}(x) u_{m}^{\prime}(y) \ln (|x-y|) d y d x \geq C W_{m} \int_{0}^{C\left\|u_{m}\right\|_{L^{\infty}}} \ln (t) d t \rightarrow 0 \quad \text { as } \quad W_{m} \rightarrow 0,
$$

as one can see by (2.10) and the argument following it in Step I of the proof of Lemma 2.3.

\section{Relaxation of the ENERGy}

We aim at proving existence of classically admissible minimizers of the energy functional (2.1) using the direct method of the calculus of variations. As we will see, the bound on the supports of low-energy profiles obtained in Section 2 is the main ingredient to obtain compactness of low-energy sequences. Since the set of classically admissible profiles is a subset of $W^{1, \infty}$ which is not even closed with respect to uniform convergence, this compactness will be established in the closure $\overline{\mathcal{A}}$ of $\mathcal{A}$ with respect to uniform convergence. In turn, we will "relax" the problem by assigning a finite relaxed energy to all functions in $\overline{\mathcal{A}}$, we will show existence of minimizers of the relaxed problem, and we will deduce existence of classically admissible minimizers for the original problem. Note that the closure of $\mathcal{A}$ with respect to the uniform convergence (in the sense of the definition (3.1) below) is the set

$$
\overline{\mathcal{A}}:=\left\{u \in W^{1, \infty}(\mathbb{R}): \operatorname{supp}(u)=[0, W], \arctan \left(u^{\prime}\right)+\theta_{m} \in\left[n^{-} \theta, n^{+} \theta\right] \text { a.e. in }(0, W)\right\}
$$

(recall that $\mathcal{N}:=\left\{n \in \mathbb{Z}, n^{-} \leq n \leq n^{+}\right\}$). To functions $u \in \overline{\mathcal{A}}$ we assign the standard "inf of the liminf" relaxed version of the energy, that is,

$$
\bar{E}(u):=\inf \left\{\varliminf_{j \rightarrow \infty} E\left(u_{j}\right): u_{j} \in \mathcal{A}, u_{j} \rightarrow u \text { uniformly, } \sup _{j \in \mathbb{N}} W_{j}<+\infty\right\} .
$$

In the above definition we could also add the requirement that the mass $\left|S\left(u_{j}\right)\right|$ of every element of the sequence $\left\{u_{j}\right\}$ is the same as the mass $|S(u)|$ of $u$. We note that the two definitions are equivalent since by the uniform bound on the supports, uniform convergence implies $L^{1}$ convergence and hence convergence of the volumes.

We will demonstrate in the sequel that in the present setting, the relaxation technique works perfectly: Precisely, for every classically admissible $u \in \mathcal{A}$ one has $\bar{E}(u)=E(u)$ (see Theorem 3.5), for every mass $m>0$ there exist relaxed energy minimizers and, finally, every minimizer of the relaxed energy is actually a classically admissible profile (see Theorem A in next Section).

We will consider separately the relaxation of the elastic energy and of the surface energy. Define $\overline{E_{\mathrm{r}}}$ and $\overline{E_{\mathrm{S}}}$ as the relaxations in the sense of (3.1) of the elastic and the surface energy, 
respectively. We will see that $\bar{E}=\overline{E_{\mathrm{r}}}+\overline{E_{\mathrm{S}}}$, but notice that this is not necessarily true in general (only one inequality is trivially always true). We start by considering the case of the elastic energy, which is slightly easier, and the relaxation result is the following.

Lemma 3.1. Let $f \in L_{\text {loc }}^{1}\left(\mathbb{R}^{+}\right)$be an increasing and concave function. Recall that for $u_{j} \in \mathcal{A}$ we denote its support by $\left[0, W_{j}\right]$. Then, for every sequence $\left\{u_{j}\right\} \subset \overline{\mathcal{A}}$ such that $\left\{W_{j}\right\}$ is bounded and $u_{j} \rightarrow u$ uniformly, one has

$$
\int_{\mathbb{R}} \int_{\mathbb{R}} u_{j}^{\prime}(y) u_{j}^{\prime}(x) f(|x-y|) d x d y \rightarrow \int_{\mathbb{R}} \int_{\mathbb{R}} u^{\prime}(y) u^{\prime}(x) f(|x-y|) d x d y .
$$

In particular, for every $u \in \overline{\mathcal{A}}$ one has

$$
\overline{E_{r}}(u)=\int_{\mathbb{R}} \int_{\mathbb{R}} u^{\prime}(y) u^{\prime}(x) \ln (|x-y|) d x d y .
$$

Proof. Since (3.3) immediately follows from (3.2) by taking $f(t)=\ln (t)$, we just have to prove (3.2), and we will do it in two steps.

Step I. The validity of (3.2) when $f$ is Lipschitz.

Consider the case in which $f$ is Lipschitz. Let $W_{j} \leq \bar{W}$ for every $j \in \mathbb{N}$. For every $y \in[0, \bar{W}]$ one has that $x \mapsto f(|x-y|)$ is continuous and bounded. Since the functions $u_{j}$ are uniformly Lipschitz by construction, we have that $u_{j} \rightarrow u$ weakly in $W^{1,2}(0, \bar{W})$ and, in particular, $u_{j}^{\prime} \longrightarrow u^{\prime}$ in $L^{2}$. Consequently, if we define

$$
\tau(y):=\int_{\mathbb{R}} u^{\prime}(x) f(|x-y|) d x, \quad \tau_{j}(y):=\int_{\mathbb{R}} u_{j}^{\prime}(x) f(|x-y|) d x,
$$

then $\tau_{j} \rightarrow \tau$ pointwise. We claim now that the functions $\tau-\tau_{j}$ are uniformly Lipschitz. Indeed, for all $y \in \mathbb{R}$ and $\varepsilon>0$,

$$
\begin{aligned}
\left|\frac{\left(\tau-\tau_{j}\right)(y+\varepsilon)-\left(\tau-\tau_{j}\right)(y)}{\varepsilon}\right| & =\frac{1}{\varepsilon}\left|\int_{\mathbb{R}}(f(|x-y-\varepsilon|)-f(|x-y|))\left(u^{\prime}(x)-u_{j}^{\prime}(x)\right) d x\right| \\
& \leq \int_{\mathbb{R}}\left|\frac{f(|x-y-\varepsilon|)-f(|x-y|)}{\varepsilon}\right|\left(\left|u^{\prime}(x)\right|+\left|u_{j}^{\prime}(x)\right|\right) d x \leq M
\end{aligned}
$$

where $M$ depends on $\bar{W}$ and on the Lipschitz constant of $f$. Therefore, the pointwise convergence of $\tau_{j}$ to $\tau$ actually implies that $\tau_{j} \rightarrow \tau$ strongly in $L^{2}$. Hence, recalling again that $u_{j}^{\prime} \longrightarrow u^{\prime}$ weakly in $L^{2}$, we have

$$
\begin{aligned}
\int_{\mathbb{R}} \int_{\mathbb{R}} u_{j}^{\prime}(y) u_{j}^{\prime}(x) f(|x-y|) d x d y & =\int_{\mathbb{R}} u_{j}^{\prime}(y) \tau_{j}(y) d y \longrightarrow \int_{\mathbb{R}} u^{\prime}(y) \tau(y) d y \\
& =\int_{\mathbb{R}} \int_{\mathbb{R}} u^{\prime}(y) u^{\prime}(x) f(|x-y|) d x d y
\end{aligned}
$$

and (3.2) follows.

Step II. The validity of (3.2) for a generic function $f$.

Consider now the case of a generic increasing and concave function $f \in L_{\mathrm{loc}}^{1}\left(\mathbb{R}^{+}\right)$, and for any $\varepsilon>0$ define

$$
f_{\varepsilon}(t):= \begin{cases}f(t), & \text { if } t \geq \varepsilon \\ f(\varepsilon)+f^{\prime}(\varepsilon)(t-\varepsilon), & \text { if } t \leq \varepsilon\end{cases}
$$


Then $f_{\varepsilon}$ is a concave, increasing, $L_{\text {loc }}^{1}$ function on $\mathbb{R}^{+}$and, in addition, it is a Lipschitz function. For $v \in \overline{\mathcal{A}}$ define

$$
\Phi(v):=\iint v^{\prime}(y) v^{\prime}(x) f(|x-y|) d x d y, \quad \Phi^{\varepsilon}(v):=\iint v^{\prime}(y) v^{\prime}(x) f_{\varepsilon}(|x-y|) d x d y .
$$

Notice that

$$
\begin{aligned}
\left|\Phi^{\varepsilon}(v)-\Phi(v)\right| & =\left|\iint v^{\prime}(y) v^{\prime}(x)\left(f_{\varepsilon}(|x-y|)-f(|x-y|)\right) d x d y\right| \\
& \leq \int_{0}^{W} \int_{y-\varepsilon}^{y+\varepsilon}\left|v^{\prime}(y)\right|\left|v^{\prime}(x)\right|\left|f(|x-y|)-f_{\varepsilon}(|x-y|)\right| d x d y \\
& \leq C W\left|\int_{0}^{\varepsilon} f(|t|) d t\right|=: C W A_{\varepsilon} .
\end{aligned}
$$

Therefore, if $\left\{u_{j}\right\}$ is a sequence as in our claim, then for every $\varepsilon>0$ one has

$$
\begin{aligned}
\varlimsup_{j \rightarrow \infty}\left|\Phi\left(u_{j}\right)-\Phi(u)\right| & \leq \varlimsup_{j \rightarrow \infty}\left[\left|\Phi\left(u_{j}\right)-\Phi^{\varepsilon}\left(u_{j}\right)\right|+\left|\Phi^{\varepsilon}\left(u_{j}\right)-\Phi^{\varepsilon}(u)\right|+\left|\Phi^{\varepsilon}(u)-\Phi(u)\right|\right] \\
& \leq 2 C \bar{W} A_{\varepsilon}+\varlimsup_{j \rightarrow \infty}\left|\Phi^{\varepsilon}\left(u_{j}\right)-\Phi^{\varepsilon}(u)\right|=2 C \bar{W} A_{\varepsilon},
\end{aligned}
$$

where we have applied the result of Step I to the Lipschitz functions $f_{\varepsilon}$. Since now $A_{\varepsilon} \searrow 0$ for $\varepsilon \searrow 0$ because $f \in L_{\text {loc }}^{1}$, the claim follows.

Remark 3.2. In the above lemma, we have used a generic increasing and concave function $f$, instead of the logarithm, just for convenience of notation. More generally, it can be easily observed that most of the results of the present paper hold true exactly in the same way if the term $\ln (|x-y|)$ in the elastic energy is replaced by $f(|x-y|)$ for an increasing, concave, $L_{\text {loc }}^{1}$ function.

We now discuss the relaxation of the surface energy. We introduce the convex set

$$
\mathcal{C}:=\operatorname{conv}\left(\left\{(\cos \phi, \sin \phi): \phi=-\theta_{m}+n \theta \pm 90^{\circ}, n \in \mathcal{N}\right\}\right)
$$

where by $\operatorname{conv}(M)$ we denote the convex hull of a set $M \in \mathbb{R}^{2}$, and we consider the associated Minkowski functional $|\cdot|^{*}$ defined by

$$
|v|^{*}:=\inf \{\lambda>0: v \in \lambda \mathcal{C}\}
$$

Notice that $|\cdot|^{*}$ is an anisotropic norm and, in particular, since $\mathcal{C}$ is contained in the unit ball, then $|\cdot|^{*} \geq|\cdot|$, the latter being the standard Euclidean norm. For a function $u \in \overline{\mathcal{A}}$, we recall that

$$
S(u):=\left\{(x, t) \in \mathbb{R}^{2}: 0 \leq x \leq W, 0 \leq t \leq u(x)\right\},
$$

and we call $\partial^{+} S(u)$ the "upper boundary" of the body, that is,

$$
\partial S^{+}(u):=\{(x, t) \in \partial S(u): t>0\}=\partial S(u) \backslash([0, W] \times\{0\}) .
$$

Denote by $\nu(x)$ the unit (in the Euclidean sense) outer normal vector at a point $x \in \partial^{+} S(u)$. 
Proposition 3.3. If $u \in \overline{\mathcal{A}}$ then

$$
\overline{E_{S}}(u)=\int_{\partial^{+} S(u)}|\nu(x)|^{*} d \mathcal{H}^{1}(x)-W .
$$

Observe that, by definition, $|\nu+\pi / 2|^{*} \geq|\nu+\pi / 2|$ for any direction $\nu \in \mathbb{S}^{1}$, and the equality holds if and only if $\nu=-\theta_{m}+n \theta$ for some $n \in \mathcal{N}$. As an immediate consequence of this observation and of the result of the above proposition, we have that for $u \in \overline{\mathcal{A}}$

$$
\overline{E_{\mathrm{S}}}(u) \geq \int_{\partial^{+} S(u)}|\nu(x)| d \mathcal{H}^{1}(x)-W,
$$

and the equality holds if and only if $u \in \mathcal{A}$. In particular, if $u \in \mathcal{A}$ then $\overline{E_{\mathrm{S}}}(u)=E_{\mathrm{S}}(u)$.

Before proving the proposition, we need first to introduce some notation and a technical auxiliary result. For $\alpha \in \mathbb{S}^{1}$ such that $-\theta_{m}+n^{-} \theta \leq \alpha \leq-\theta_{m}+n^{+} \theta$, we set

$$
\alpha^{-}:=\max \left\{\phi \leq \alpha: \phi=\theta_{m}+n \theta, n \in \mathcal{N}\right\}, \quad \alpha^{+}:=\min \left\{\phi \geq \alpha: \phi \theta_{m}+n \theta, n \in \mathcal{N}\right\} .
$$

Of course, $\alpha^{+}=\alpha^{-}+\theta$ unless $\alpha^{+}=\alpha^{-}=\alpha$, which in turn happens if and only if $\alpha=-\theta_{m}+n \theta$ for some $n \in \mathcal{N}$. Moreover, for $\nu \in \mathbb{S}^{1}$ we denote $|\nu|_{*}:=\left|\nu+\frac{\pi}{2}\right|^{*}$, and for a Lipschitz curve $\gamma$ (parametrized by arc-length) satisfying $-\theta_{m}+n^{-} \theta \leq \arctan \gamma^{\prime}(t) \leq-\theta_{m}+n^{+} \theta$ for a.e. $t$, we define its "length" as

$$
\operatorname{length}_{*}(\gamma):=\int\left|\gamma^{\prime}(t)\right|_{*} d t
$$

In particular, the length ${ }_{*}$ of the graph of a function $u \in \overline{\mathcal{A}}$ is greater than the Euclidean length of the same graph, and the equality holds if and only if $u \in A$. We are now ready to state and prove our technical result.

Lemma 3.4. Let $A, B \in \mathbb{R}^{2}$ be such that $\alpha:=\frac{B-A}{|B-A|} \in \mathbb{S}^{1}$ belongs to $\left(-\theta_{m}+n^{-} \theta,-\theta_{m}+n^{+} \theta\right)$, and let $\gamma$ be a Lipschitz curve connecting $A$ and $B$ with $-\theta_{m}+n^{-} \theta \leq \arctan \gamma^{\prime}(t) \leq-\theta_{m}+n^{+} \theta$ for a.e. $t$. Then

$$
\text { length }_{*}(\gamma) \geq|B-A| \frac{\cos \left(\frac{\alpha^{+}+\alpha^{-}}{2}-\alpha\right)}{\cos \left(\frac{\alpha^{+}-\alpha^{-}}{2}\right)},
$$

and the equality holds if and only if $\gamma^{\prime}(t) \in\left[\alpha^{-}, \alpha^{+}\right]$for a.e. $t$.

Proof. Up to a rotation, we can reduce to the case when $\alpha^{-}=0$. Notice that this rotation implies in particular that for horizontal lines, i.e., lines with slope 0 , the length $\operatorname{t*}_{(*)} \mathrm{s}$ given by $|\cdot|_{*}$ and $|\cdot|$ coincide. If also $\alpha^{+}=0$, then it must be $\alpha=0$, so the formula to prove is simply length $_{*}(\gamma) \geq|B-A|$, which is true because $|\cdot|_{*} \geq|\cdot|$, and the formula is an equality if and only if $\gamma$ is the segment connecting $A$ and $B$.

Therefore, it suffices to consider the case when $\alpha^{+}=\theta$. We observe that all curves connecting $A$ and $B$ and whose direction is always between 0 and $\theta$ have the same length . This is a well known property of the anisotropic length, and it directly comes from the definition. An elementary trigonometric calculation ensures that the common length of all these curves is precisely given by the right formula in (3.7). Therefore, the proof of the lemma will follow once 
we prove the following

Claim. For every Lipschitz curve $\gamma$ as in the claim connecting $A$ and $B$, there exists another Lipschitz curve $\gamma_{\infty}$, still connecting $A$ and $B$, having always direction between 0 and $\theta$, and such that length ${ }_{*}\left(\gamma_{\infty}\right) \leq$ length $_{*}(\gamma)$. Moreover, the inequality is strict unless the direction of $\gamma$ is always between 0 and $\theta$.

We divide the remaining of the proof in two steps.

Step I. Construction of $\tilde{\beta}$ from $\beta$ and its properties.

Let $\beta$ be a path connecting two points $C$ and $D$, and such that the direction of the segment $C D$ is between 0 and $\theta$. Consider, as in Figure 6, the four lines having directions 0 and $\theta$, and passing through $C$ and $D$, which determine a (closed) parallelogram $\mathcal{T}$. Our aim is to define a path $\tilde{\beta}$ connecting $C$ and $D$, entirely contained in $\mathcal{T}$, and such that either $\tilde{\beta}=\beta$, or $\operatorname{length}_{*}(\tilde{\beta})<\operatorname{length}_{*}(\beta)$.

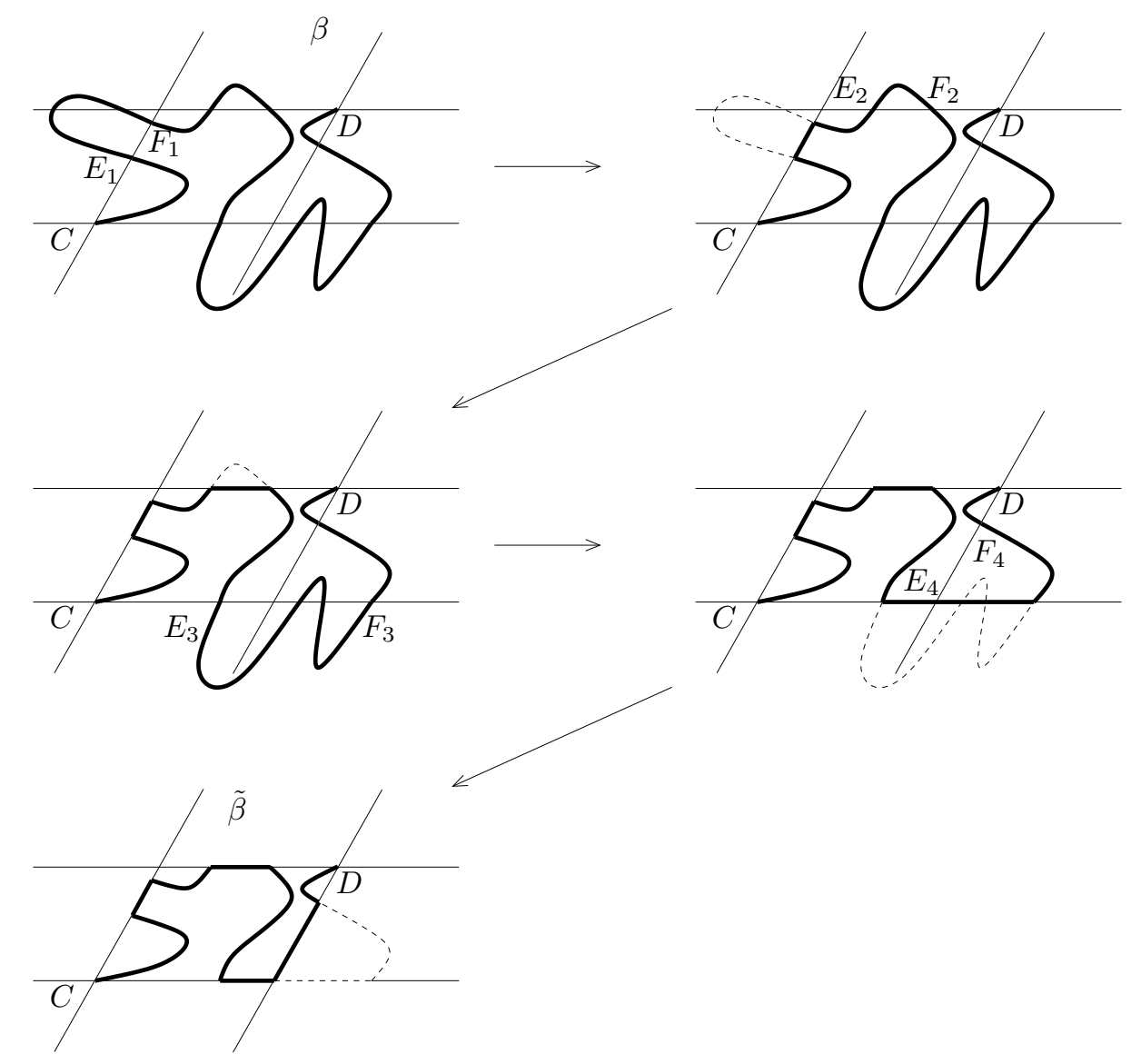

Figure 6. Construction of $\tilde{\beta}$ from $\beta$ in Lemma 3.4 .

If $\beta$ is entirely contained in $\mathcal{T}$, then we define $\tilde{\beta}:=\beta$, and the step is concluded. Otherwise, let $E_{1}$ (possibly $C$ itself) be the last point of $\beta$ such that the whole subpath of $\beta$ from $C$ to $E_{1}$ is contained in $\mathcal{T}$. Hence, $E_{1}$ is on one of the four lines that we are considering and after $E_{1}$, at least for a while, the path will be on the halfspace delimited by this line and not containing $\mathcal{T}$. We define then $F_{1}$ (possibly $D$ itself) the last point of $\beta$ in this halfspace, so that $E_{1}$ and 
$F_{1}$ are on a same line; hence, we modify $\beta$ by removing the whole part between $E_{1}$ and $F_{1}$ and substituting it with the segment. This process strictly decreases the length ${ }_{*}$, because the Euclidean length of the segment $E_{1} F_{1}$ is strictly less than the Euclidean length of the part of the path that we have removed, which in turn is less than the length ${ }_{*}$ of the same part. On the other hand, for the segment $E_{1} F_{1}$ the Euclidean length and the length ${ }_{*}$ coincide by definition, since the direction of the segment (either 0 or $\theta$ ) is one of the "good" directions. If this new path is entirely contained in $\mathcal{T}$ we call it $\tilde{\beta}$ and the step is concluded, otherwise we do the same procedure again, and this will "rule out" a second line, different from the first one. At most after four steps, then (which is the case depicted in the Figure), the resulting path is entirely contained in $\mathcal{T}$, and its length has been stricly decreased at every step. We call this path $\tilde{\beta}$ and the step is then concluded.

Step II. Proof of the Claim.

We can now prove the Claim and, as discussed above, this will conclude also the proof of the lemma.

We start by applying the construction of Step I to the whole path $\beta=\gamma$, then with $C=A$ and $D=B$, and we define $\gamma_{1}:=\tilde{\beta}$. The path $\gamma_{1}$ is still a path connecting $A$ and $B$, and it is entirely contained in the parallelogram of directions 0 and $\theta$ with opposite corners at $A$ and $B$.

Set $L_{1}:=\operatorname{length}_{*}\left(\gamma_{1}\right)$, so that the arc-length parametrization of the path is $\gamma_{1}:\left[0, L_{1}\right] \rightarrow \mathbb{R}^{2}$. We apply twice the construction of Step I, once being $\beta$ the restriction of $\gamma_{1}$ to the interval $\left[0, L_{1} / 2\right]$, and once being $\beta$ the restriction to the interval $\left[L_{1} / 2, L_{1}\right]$, and we define $\gamma_{2}$ to be the union of the two resulting paths $\tilde{\beta}$. Thus $\gamma_{2}$ is another path between $A$ and $B$, and not only it is contained in the parallelogram determined by $A$ and $B$, but also in the union of the two parallelograms determined by $A$ and $\gamma_{1}\left(L_{1} / 2\right)$, and by $\gamma_{1}\left(L_{1} / 2\right)$ and $B$, respectively.

We continue our definition recursively, obtaining from each path $\gamma_{j}$ the next path $\gamma_{j+1}$ dividing the interval of definition in $2^{j}$ equal parts. It is immediate to observe that the sequence of Lipschitz paths $\gamma_{j}$ is uniformly converging to a Lipschitz path $\gamma_{\infty}$, as well as that $\arctan \left(\gamma_{\infty}^{\prime}(t)\right)$ is between 0 and $\theta$ almost everywhere. Since every step has decreased the length, it is clear that $\operatorname{length}_{*}\left(\gamma_{\infty}\right) \leq \operatorname{length}_{*}(\gamma)$. Moreover, every single step, say the $j$-th, has strictly decreased the length ${ }_{*}$, unless $\gamma_{j}=\gamma_{j-1}$. As a consequence, either length ${ }_{*}\left(\gamma_{\infty}\right)<\operatorname{length}_{*}(\gamma)$, or $\gamma_{\infty}=\gamma$ which, in turn, holds if and only if the direction of the original path $\gamma$ was already always between 0 and $\theta$.

We can now finally prove Proposition 3.3.

Proof (of Proposition 3.3). Recall that for every classically admissible function $u \in \mathcal{A}$ we have by definition $|\nu(x)|^{*}=1$ for $\mathcal{H}^{1}$-a.e. point $x \in \partial^{+} S(u)$, hence

$$
E_{\mathrm{S}}(u)=\operatorname{length}(\operatorname{Graph}(u))-W=\mathcal{H}^{1}\left(\partial^{+} S(u)\right)-W=\int_{\partial^{+} S(u)}|\nu(x)|^{*} d \mathcal{H}^{1}(x)-W .
$$


Therefore, establishing the claim reduces to proving that, for every $u \in \overline{\mathcal{A}}$,

$$
\begin{aligned}
\int_{\partial^{+} S(u)}|\nu(x)|^{*} d \mathcal{H}^{1}-W=\inf \left\{\varliminf_{j \rightarrow \infty} \int_{\partial^{+} S\left(u_{j}\right)}|\nu(x)|^{*} d \mathcal{H}^{1}-W_{j}:\right. \\
\left.u_{j} \in \mathcal{A}, u_{j} \rightarrow u \text { uniformly, } \sup _{j \in \mathbb{N}} W_{j}<+\infty\right\} .
\end{aligned}
$$

In view of Lemma 3.4, we work with paths, instead of functions. Therefore, let $\bar{\Gamma}$ be the set of Lipschitz paths in $\mathbb{R}^{2}$ having always direction between $-\theta_{m}+n^{-} \theta$ and $-\theta_{m}+n^{+} \theta$, and let $\Gamma$ be the set of Lipschitz paths whose direction is always of the form $-\theta_{m}+n \theta$ for some $n \in \mathcal{N}$. We will prove that for every $\gamma \in \bar{\Gamma}$

$$
\operatorname{length}_{*}(\gamma)=\inf \left\{\varliminf_{j \rightarrow \infty} \operatorname{length}_{*}\left(\gamma_{j}\right): \gamma_{j} \in \Gamma, \gamma_{j} \rightarrow \gamma \text { uniformly }\right\} .
$$

Since the length ${ }_{*}$ is the length associated to a convex norm, it is well-known that whenever $\gamma_{j} \rightarrow \gamma$ uniformly then length ${ }_{*}(\gamma) \leq \underline{\lim }_{j \rightarrow \infty} \operatorname{length}_{*}\left(\gamma_{j}\right)$, for every $\gamma$ and $\gamma_{j}$ even in the larger space $\bar{\Gamma}$. Hence, it suffices to consider a generic path $\gamma \in \bar{\Gamma}$ and to find a "recovery sequence", that is, a sequence $\left\{\gamma_{j}\right\}$ contained in $\Gamma$, uniformly converging to $\gamma$ and with length ${ }_{*}\left(\gamma_{j}\right) \rightarrow \operatorname{length}_{*}(\gamma)$.

Define, for every $j \in \mathbb{N}, \gamma^{j}$ as the piecewise affine path connecting all the points $\gamma(i / j)$ for $0 \leq i \leq j$. Then $\gamma^{j} \in \bar{\Gamma}, \gamma^{j} \rightarrow \gamma$ uniformly when $j \rightarrow \infty$, and $\operatorname{length}_{*}\left(\gamma^{j}\right) \leq \operatorname{length}_{*}(\gamma)$. Fix $0 \leq i<j$ and consider the part of the path $\gamma^{j}$ which is the segment between $\gamma(i / j)$ and $\gamma((i+1) / j)$. This is a segment with direction $-\theta_{m}+n^{-} \theta \leq \alpha \leq-\theta_{m}+n^{+} \theta$, so we can define $\alpha^{-}$and $\alpha^{+}$as in (3.6). Replace the segment with two segments, one with direction $\alpha^{-}$ and the other with direction $\alpha^{+}$, still connecting $\gamma(i / j)$ with $\gamma((i+1) / j$ ) (in particular, we do not change anything if $\left.\alpha^{-}=\alpha^{+}\right)$. This path $\gamma_{j}$ obtained from $\gamma^{j}$ is such that $\gamma_{j} \in \Gamma$ and $\operatorname{length}_{*}\left(\gamma_{j}\right)=\operatorname{length}_{*}\left(\gamma^{j}\right)$ by Lemma 3.4. Since the new sequence $\left\{\gamma_{j}\right\}$ converges uniformly to $\gamma$, we have that

$$
\varliminf_{j \rightarrow \infty} \operatorname{length}_{*}\left(\gamma_{j}\right)=\varliminf_{j \rightarrow \infty} \operatorname{length}_{*}\left(\gamma^{j}\right) \leq \operatorname{length}_{*}(\gamma) .
$$

As the opposite inequality is always true, the above estimate is actually an equality and so $\left\{\gamma_{j}\right\}$ is the required recovery sequence, and the proof of (3.9) is concluded.

We now conclude the proof of the Proposition, namely, (3.8). First, let $\left\{u_{j}\right\} \subseteq \mathcal{A}$ be any sequence with $\left\{W_{j}\right\}$ bounded, and uniformly converging to $u \in \overline{\mathcal{A}}$. Up to a subsequence, we may assume that $W_{j} \underset{j \rightarrow \infty}{\longrightarrow} \widetilde{W} \geq W$. Let $\gamma_{j}$ be the Lipschitz path corresponding to $\partial S^{+}\left(u_{j}\right)$, and notice that $\left\{\gamma_{j}\right\}$ uniformly converges to $\gamma$, where $\gamma$ is not the path corresponding to $\partial S^{+}(u)$, but this path plus the segment $\sigma:=[W, \widetilde{W}] \times\{0\}$ (clearly, if $\widetilde{W}=W$ then $\sigma$ is an empty segment, so actually $\gamma$ is the path corresponding to $\left.\partial S^{+}(u)\right)$. Thanks to $(3.9)$, we have

$$
\begin{aligned}
\int_{\partial^{+} S(u)}|\nu(x)|^{*} d \mathcal{H}^{1}-W & =\operatorname{length}_{*}(\gamma)-\operatorname{length}_{*}(\sigma)-W \leq \operatorname{length}_{*}(\gamma)-\widetilde{W} \\
& \leq \varliminf_{j \rightarrow \infty} \operatorname{length}_{*}\left(\gamma_{j}\right)-W_{j}=\varliminf_{j \rightarrow \infty} \int_{\partial^{+} S\left(u_{j}\right)}|\nu(x)|^{*} d \mathcal{H}^{1}-W_{j},
\end{aligned}
$$

and hence the first inequality in (3.8) follows. To conclude, we need to find a recovery sequence for (3.8). Let $\gamma$ be the Lipschitz path corresponding to $\partial S^{+}(u)$ for a generic $u \in \overline{\mathcal{A}}$, let $\left\{\gamma_{j}\right\}$ be 
the recovery sequence found above, and let $u_{j} \in \mathcal{A}$ be the function corresponding to each $\gamma_{j}$. We have proved above that

$$
\int_{\partial^{+} S\left(u_{j}\right)}|\nu(x)|^{*} d \mathcal{H}^{1}=\operatorname{length}_{*}\left(\gamma_{j}\right) \underset{j \rightarrow \infty}{\longrightarrow} \operatorname{length}_{*}\left(\gamma_{j}\right)=\int_{\partial^{+} S(u)}|\nu(x)|^{*} d \mathcal{H}^{1},
$$

and notice that $W_{j}=W$ for every $j$ by construction. Hence $\left\{u_{j}\right\}$ is the recovery sequence, and this concludes the proof of (3.8).

Summarizing, we have the following result.

Theorem 3.5. The relaxation $\bar{E}$ of $E$ coincides with $\overline{E_{r}}+\overline{E_{s}}$, where for $u \in \overline{\mathcal{A}}$

$$
\overline{E_{r}}(u):=\int_{0}^{W} \int_{0}^{W} u^{\prime}(x) u^{\prime}(y) \ln (|x-y|) d x d y, \quad \overline{E_{s}}(u):=\int_{\partial^{+} S(u)}|\nu(x)|^{*} d \mathcal{H}^{1}-W,
$$

and $\nu(x)$ denotes the unit normal vector to $S(u)$ at $u(x)$. In particular, if $u \in \mathcal{A}$ then $\overline{E_{r}}(u)=$ $E_{r}(u)$ and $\overline{E_{s}}(u)=E_{s}(u)$.

\section{Theorem A: eXistence of Classical Minimizers}

In this section we prove that there exist classical minimizers for every positive mass. For that, we first show that there exist minimizers of the relaxed energy functional and then establish that every minimizer of the relaxed problem is in fact classical. Recall that the classically admissible slopes play a decisive role in the relaxed surface energy (see Theorem 3.5, (3.4) and (3.5)). Consequently, to show that minimizers of the relaxed problem are classical, we will make use of the anisotropy of the relaxed surface energy dictated by the admissible slopes $-\theta_{m}+n \theta, n \in \mathcal{N}$ (see Subsection 2.1).

Theorem A (Existence of classical minimizers). For any mass $m>0$ there exist minimizers $u \in \overline{\mathcal{A}}$ of the relaxed energy functional $\bar{E}$. Moreover, every minimizer of $\bar{E}$ is an element of $\mathcal{A}$, thus it is also a classical minimizer of the original energy functional $E$.

Proof. We start by proving the existence of relaxed minimizers. Fix $m>0$, and let $\left\{u_{j}\right\}$ be a minimizing sequence of $\bar{E}$ in $\overline{\mathcal{A}}$. By the definition (3.1) of the relaxation, we may assume that every $u_{j}$ belongs to $\mathcal{A}$. In view of Proposition 2.4, we have that the widths $W_{j}$ are uniformly bounded, and, up to a subsequence (not relateled) the sequence $\left\{u_{j}\right\}$ converges uniformly to some $u \in \overline{\mathcal{A}}$. Clearly $u$ has mass $m$, and since by definition the relaxed energy is lower semicontinuous, we conclude that $\bar{E}(u) \leq \underline{\lim }_{j \rightarrow \infty} \bar{E}\left(u_{j}\right)=\mathcal{F}(m)$. In other words, $u$ is a relaxed minimizer.

To conclude the proof, we have to show that every relaxed minimizer is in fact classical, i.e., it belongs to $\mathcal{A}$. Assume, by contradiction, that $u \in \overline{\mathcal{A}} \backslash A$ is a minimizer with mass $m$. Then there is some point $\bar{x} \in(0, W)$ which is a Lebesgue point for $u^{\prime}$ satisfying

$$
\arctan \left(u^{\prime}(\bar{x})\right) \in\left(\theta_{i}, \theta_{i+1}\right),
$$

for some $n^{-} \leq i<n^{+}$, with $\theta_{i}:=\theta_{m}+i \theta$ for every $i \in \mathcal{N}$. Define

$$
\eta:=\min \left\{\theta_{i+1}-u^{\prime}(\bar{x}), u^{\prime}(\bar{x})-\theta_{i}\right\},
$$


and let $K \gg 1$ be a number to be fixed later. By the definition of Lebesgue points, for $\varepsilon>0$ small enough we have

$$
\frac{1}{2 \varepsilon} \int_{\bar{x}-\varepsilon}^{\bar{x}+\varepsilon}\left|u^{\prime}(x)-u^{\prime}(\bar{x})\right| d x<\frac{\eta}{2 K},
$$

and this implies that

$$
\frac{\eta}{2 K}>\frac{1}{2 \varepsilon} \int_{\bar{x}-\varepsilon}^{\bar{x}+\varepsilon}\left|u^{\prime}(x)-u^{\prime}(\bar{x})\right| d x \geq \frac{\eta}{2 \varepsilon}\left|\left\{x \in(\bar{x}-\varepsilon, \bar{x}+\varepsilon):\left|u^{\prime}(x)-u^{\prime}(\bar{x})\right|>\eta\right\}\right| .
$$

Hence, if we define the set of "bad" points as

$$
B:=\left\{x \in(\bar{x}-\varepsilon, \bar{x}+\varepsilon):\left|u^{\prime}(x)-u^{\prime}(\bar{x})\right|>\eta\right\},
$$

we have $|B|<\varepsilon / K$. We call the set of "good" points $G:=(\bar{x}-\varepsilon, \bar{x}+\varepsilon) \backslash B$.

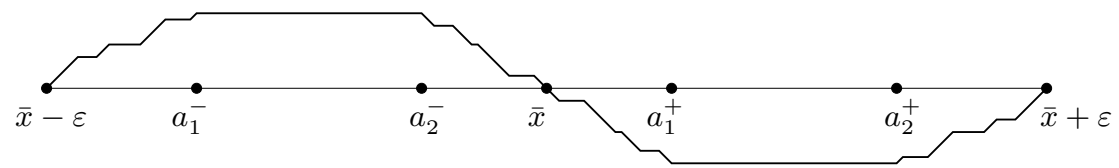

FIGURE 7. Schematic sketch of the variation $\varphi$

We will now use the anisotropy of the relaxed surface energy to build a comparison function, which has the same surface energy but lower elastic energy. First pick four points $a_{1}^{ \pm}, a_{2}^{ \pm}$ satisfying

$$
\bar{x}-\varepsilon<a_{1}^{-}<\bar{x}-\varepsilon+\frac{\varepsilon}{K}<\bar{x}-\frac{\varepsilon}{K}<a_{2}^{-}<\bar{x}<a_{1}^{+}<\bar{x}+\frac{\varepsilon}{K}<\bar{x}+\varepsilon-\frac{\varepsilon}{K}<a_{2}^{+}<\bar{x}+\varepsilon
$$

and with the property that

$$
0<\left|G \cap\left(\bar{x}-\varepsilon, a_{1}^{-}\right)\right|=\left|G \cap\left(a_{2}^{-}, \bar{x}\right)\right|, \quad 0<\left|G \cap\left(\bar{x}, a_{1}^{+}\right)\right|=\left|G \cap\left(a_{2}^{+}, \bar{x}+\varepsilon\right)\right| .
$$

Define now the function $\varphi \in W_{0}^{1, \infty}((\bar{x}-\varepsilon, \bar{x}+\varepsilon))$ by (see Figure 7 )

$$
\varphi^{\prime}(x):=\left\{\begin{aligned}
1, & \text { if } x \in G \cap\left(\left(\bar{x}-\varepsilon, a_{1}^{-}\right) \cup\left(a_{2}^{+}, \bar{x}+\varepsilon\right)\right), \\
-1, & \text { if } x \in G \cap\left(a_{2}^{-}, a_{1}^{+}\right), \\
0 & \text { otherwise. }
\end{aligned}\right.
$$

By definition, $\varphi$ is continuous and $\varphi(\bar{x}-\varepsilon)=\varphi(\bar{x})=\varphi(\bar{x}+\varepsilon)=0$. Moreover, up to a small change in $a_{1}^{ \pm}$and $a_{2}^{ \pm}$, we may assume that, in addition,

$$
\int_{\bar{x}-\varepsilon}^{\bar{x}+\varepsilon} \varphi(x) d x=0 \text {. }
$$

Define now, for every $-\eta<\delta<\eta$, the new function $u_{\delta}:=u+\delta \varphi$. Observe that $u_{\delta}=u$ outside of the interval $(\bar{x}-\varepsilon, \bar{x}+\varepsilon)$, and in this interval $u_{\delta}^{\prime}(x)=u^{\prime}(x)$ if $x \in B$ while, if $x \in G$, then $u_{\delta}^{\prime}(x)$ can be equal to either $u^{\prime}(x)$, or $u^{\prime}(x)-\delta$, or $u^{\prime}(x)+\delta$. In any case, by the definition of $G$ and $B$, it is $u_{\delta}^{\prime}(x) \in\left(\theta_{i}, \theta_{i+1}\right)$ for every $x \in G$. As a consequence, we obtain that $u_{\delta} \in \overline{\mathcal{A}}$, and 
moreover $\left|S\left(u_{\delta}\right)\right|=|S(u)|$ thanks to (4.1). In particular, recalling Lemma 3.4, we obtain that for every $-\eta<\delta<\eta$ it is $\overline{E_{\mathrm{S}}}\left(u_{\delta}\right)=\overline{E_{\mathrm{S}}}(u)$.

Concerning the elastic energy, if $\varepsilon$ is small enough and $K$ is big enough then $\overline{E_{\mathrm{r}}}(\varphi)<0$. As a consequence, we have

$$
\begin{aligned}
\bar{E}\left(u_{\delta}\right)-\bar{E}(u) & =\overline{E_{\mathrm{r}}}\left(u_{\delta}\right)-\overline{E_{\mathrm{r}}}(u) \\
& =\delta \int_{0}^{W} \int_{0}^{W}\left(u^{\prime}(x) \varphi^{\prime}(y)+u^{\prime}(y) \varphi^{\prime}(x)\right) \ln (|x-y|) d y d x+\delta^{2} \overline{E_{\mathrm{r}}}(\varphi) .
\end{aligned}
$$

Therefore, there exist some $\delta \in(-\eta, \eta)$ such that $\bar{E}\left(u_{\delta}\right)<\bar{E}(u)$, which is a contradiction because $u$ was assumed to be optimal.

\section{Estimates For Rescaled PROfiles}

This auxiliary section is devoted to the study of what happens to the admissible profiles under rescaling. Note that the scaling properties are not specific about this model but typical for various atomistic and continuum models for epitaxial growth. As typical in models for pattern formation, the surface and the elastic energy scale differently with the volume, and consequently, different contributions dominate in different regimes. When rescaling admissible profile functions, one has to be careful to respect the slope constraint. The scaling results and its consequences obtained here closely parallel results for continuum models without miscut angle and slope constraint obtained in [1]. From this analysis, the concavity of the function $m \mapsto \mathcal{F}(m)$ directly follows, while other properties will be obtained in the next sections.

Let us start by defining the rescaled profiles: For an admissible function $u \in \overline{\mathcal{A}}$, and for any $\varepsilon>-1$, we define

$$
u_{\varepsilon}(x):=(1+\varepsilon) u\left(\frac{x}{1+\varepsilon}\right)
$$

Since

$$
u_{\varepsilon}^{\prime}(x)=u^{\prime}\left(\frac{x}{1+\varepsilon}\right),
$$

we have that $u_{\varepsilon} \in \overline{\mathcal{A}}$ and, in particular, if $u \in \mathcal{A}$ then also $u_{\varepsilon} \in \mathcal{A}$. Moreover,

$$
\left|S\left(u_{\varepsilon}\right)\right|=(1+\varepsilon)^{2}|S(u)|, \quad \overline{E_{\mathrm{S}}}\left(u_{\varepsilon}\right)=(1+\varepsilon) \overline{E_{\mathrm{S}}}(u),
$$

as well as

$$
\begin{aligned}
\overline{E_{\mathrm{r}}}\left(u_{\varepsilon}\right) & =\int_{0}^{W(1+\varepsilon)} \int_{0}^{W(1+\varepsilon)} u_{\varepsilon}^{\prime}(\tilde{x}) u_{\varepsilon}^{\prime}(\tilde{y}) \ln (|\tilde{x}-\tilde{y}|) d \tilde{x} d \tilde{y} \\
& =(1+\varepsilon)^{2} \int_{0}^{W} \int_{0}^{W} u^{\prime}(x) u^{\prime}(y) \ln (|x-y||1+\varepsilon|) d x d y \\
& =(1+\varepsilon)^{2} \int_{0}^{W} \int_{0}^{W} u^{\prime}(x) u^{\prime}(y) \ln (|x-y|)+(1+\varepsilon)^{2} \underbrace{\int_{0}^{W} \int_{0}^{W} u^{\prime}(x) u^{\prime}(y) \ln (1+\varepsilon)}_{=0} \\
& =(1+\varepsilon)^{2} \overline{E_{\mathrm{r}}}(u) .
\end{aligned}
$$


The above estimates imply that for any admissible function $u \in \overline{\mathcal{A}}$

$$
\bar{E}\left(u_{\varepsilon}\right)=(1+\varepsilon)^{2} \overline{E_{\mathrm{r}}}(u)+(1+\varepsilon) \overline{E_{\mathrm{S}}}(u) .
$$

As a first byproduct of this formula we deduce the following continuity result.

Lemma 5.1. The function $m \mapsto \mathcal{F}(m)$ is continuous.

Proof. Fix $m>0$. The lower semi-continuity of $\mathcal{F}$ is standard. For every $|\delta| \ll 1$ let $v_{\delta}$ be a minimizer of the energy for volume $m+\delta$, which exists by Theorem A. Recalling Proposition 2.4, we deduce that the widths $W\left(v_{\delta}\right)$ are uniformly bounded, thus by Ascoli-Arzelà Theorem a subsequence of $\left\{v_{\delta}\right\}$ uniformly converges to some profile $v$ of mass $m$. By the definition (3.1) of the rescaled energy, we have

$$
\mathcal{F}(m) \leq \bar{E}(v) \leq \varliminf_{\delta \rightarrow 0} \bar{E}\left(v_{\delta}\right)=\varliminf_{\delta \rightarrow 0} \mathcal{F}(m+\delta),
$$

hence the lower semi-continuity follows.

On the other hand, again in view of Theorem A, letu be an energy minimizer for mass $m$, and by (5.2) we conclude that

$$
\varlimsup_{\varepsilon \rightarrow 0} \mathcal{F}\left(m(1+\varepsilon)^{2}\right) \leq \varlimsup_{\varepsilon \rightarrow 0} \bar{E}\left(u_{\varepsilon}\right)=\varlimsup_{\varepsilon \rightarrow 0} \bar{E}(u)+\varepsilon\left(2 \overline{E_{\mathrm{r}}}(u)+\overline{E_{\mathrm{S}}}(u)+O(\varepsilon)\right)=\bar{E}(u)=\mathcal{F}(m),
$$

and so the upper semi-continuity follows.

We introduce now the following notation.

Definition 5.2. For every mass $m>0$, define

$$
\begin{aligned}
& C^{+}(m):=\sup \left\{\overline{E_{S}}(u):|S(u)|=m, \bar{E}(u)=\mathcal{F}(m)\right\}, \\
& C^{-}(m):=\inf \left\{\overline{E_{S}}(u):|S(u)|=m, \bar{E}(u)=\mathcal{F}(m)\right\} .
\end{aligned}
$$

The rescaling properties imply even more regularity of $\mathcal{F}$ (see [1] for similar results for unfaceted island growth with zero miscut).

Theorem 5.3. The function $\mathcal{F}$ is concave. Moreover, for every $m>0$ the left and the right derivative of $\mathcal{F}$ at $m$ are given by

$$
\mathcal{F}_{-}^{\prime}(m)=\frac{1}{m}\left(\mathcal{F}(m)-\frac{C^{-}(m)}{2}\right), \quad \mathcal{F}_{+}^{\prime}(m)=\frac{1}{m}\left(\mathcal{F}(m)-\frac{C^{+}(m)}{2}\right) .
$$

Proof. Notice that, by (5.1), the admissible profiles of mass $m$ are all and only the functions of the form $u_{\varepsilon}$, where $u$ is an admissible profile of mass 1 , and $(1+\varepsilon)^{2}=m$. In view of $(5.2)$, we have

$$
\begin{aligned}
\mathcal{F}(m) & =\inf \{\bar{E}(u): u \in \overline{\mathcal{A}},|S(u)|=m\}=\inf \left\{\bar{E}\left(u_{\varepsilon}\right): u \in \overline{\mathcal{A}},|S(u)|=1,(1+\varepsilon)^{2}=m\right\} \\
& =\inf \left\{m \overline{E_{\mathrm{r}}}(u)+\sqrt{m} \overline{E_{\mathrm{S}}}(u): u \in \overline{\mathcal{A}},|S(u)|=1\right\} .
\end{aligned}
$$

Since the surface energy of any admissible function is always positive, the function $m \mapsto$ $m \overline{E_{\mathrm{r}}}(u)+\sqrt{m} \overline{E_{\mathrm{S}}}(u)$ is concave for every $u \in \overline{\mathcal{A}}$ with mass 1 , and so $\mathcal{F}$ is the infimum of concave functions, hence it is concave. 
Let now $\nu_{j} \searrow 0$ be any inifinitesimal sequence, and let $u^{j}$ be energy minimizers of mass $m+\nu_{j}$. Let $\varepsilon_{j}$ be such that $\left(m+\nu_{j}\right)\left(1+\varepsilon_{j}\right)^{2}=m$. For every $j$ we have that $u_{\varepsilon_{j}}^{j}$ is a profile with mass $m$, hence

$$
\begin{aligned}
\mathcal{F}(m) & \leq \bar{E}\left(u_{\varepsilon_{j}}^{j}\right)=\frac{m}{m+\nu_{j}} \overline{E_{\mathrm{r}}}\left(u_{j}\right)+\left(\frac{m}{m+\nu_{j}}\right)^{\frac{1}{2}} \overline{E_{\mathrm{S}}}\left(u_{j}\right) \\
& =\bar{E}\left(u_{j}\right)-\frac{\nu_{j}}{m} \overline{E_{\mathrm{r}}}\left(u_{j}\right)-\frac{\nu_{j}}{2 m} \overline{E_{\mathrm{S}}}\left(u_{j}\right)+o\left(\nu_{j}\right) \\
& =\mathcal{F}\left(m+\nu_{j}\right)-\frac{\nu_{j}}{m}\left(\mathcal{F}\left(m+\nu_{j}\right)-\frac{\overline{E_{\mathrm{S}}}\left(u_{j}\right)}{2}\right)+o\left(\nu_{j}\right) .
\end{aligned}
$$

Observe now that by Proposition 2.4 the uniform boundedness of the widths $W_{j}$ follows, and thus, up to a subsequence (not relabeled), we have that $\left\{u^{j}\right\}$ uniformly converges to some $u$. The profile $u$ clearly satisfies $|S(u)|=m$, and moreover, by Lemma $3.1, \overline{E_{\mathrm{r}}}\left(u^{j}\right) \rightarrow \overline{E_{\mathrm{r}}}(u)$. Since $\mathcal{F}$ is continuous by Lemma 5.1, we deduce that $u$ is a minimizer for mass $m$, and $\overline{E_{\mathrm{S}}}\left(u^{j}\right) \rightarrow \overline{E_{\mathrm{S}}}(u)$. We pass to the limit in $j$ and deduce that

$$
\begin{aligned}
\mathcal{F}_{+}^{\prime}(m) & =\lim _{j \rightarrow \infty} \frac{\mathcal{F}\left(m+\nu_{j}\right)-\mathcal{F}(m)}{\nu_{j}} \geq \varlimsup_{j \rightarrow \infty} \frac{1}{m}\left(\mathcal{F}\left(m+\nu_{j}\right)-\frac{\overline{E_{\mathrm{S}}}\left(u_{j}\right)}{2}\right)+o(1) \\
& =\frac{1}{m}\left(\mathcal{F}(m)-\frac{\overline{E_{\mathrm{S}}}(u)}{2}\right) \geq \frac{1}{m}\left(\mathcal{F}(m)-\frac{C^{+}(m)}{2}\right) .
\end{aligned}
$$

On the other hand, if $u$ is a minimizer of mass $m$, then the same argument of (5.3) yields

$$
\mathcal{F}(m+\nu) \leq \mathcal{F}(m)+\frac{\nu}{m+\nu}\left(\mathcal{F}(m)-\frac{\overline{E_{\mathrm{S}}}(u)}{2}\right)+o(\nu)
$$

for any $\nu>0$, so we obtain the opposite inequality of (5.4), and then formula for the right derivative $\mathcal{F}_{+}^{\prime}(m)$ is established. The very same considerations with negative $\nu_{j}$ and $\nu$ yield the formula for the left derivative.

Remark 5.4. Since for a concave function the left and right derivatives coincide at all but at most countably many points, we derive that for almost all $m>0$, we have $C^{+}(m)=C^{-}(m)$. This does not imply the uniqueness of the minimizer for mass $m$, but at least it ensures that, if there are different minimizers, they must all have the same surface energy, and hence, also the same strain energy (see also [1]). Note that uniqueness of minimizers is not expected in general. We refer to [9] for a non-uniqueness result for a continuum model to describe periodic island formation without miscut and slope constraint.

\section{Theorem B: Generalized Zero Contact Angle}

As already mentioned in the Introduction, in several different models for epitaxy and the study of energy minimizers for the thin film problems, the optimal profiles start and end tangentially to the substrate, i.e., have the so-called "zero contact angle" property (see $[7,9,1]$ ). In the present model, we will prove the following "faceted" generalization of this effect. 
Theorem B (Generalized zero contact angle). If $u$ is an energy minimizing profile, then it starts with a segment of slope $\theta-\theta_{m}$ and it ends with a segment of slope $-\theta_{m}$. Moreover, the length of each of these two segments can be estimated from below in terms of the mass of the profile.

Proof. Let $u$ be an energy minimizing profile of mass $m>0$. We want to prove that $u$ ends with a segment of slope $-\theta_{m}$, that is, $\arctan \left(u^{\prime}\right) \equiv-\theta_{m}$ on an interval of the form $(W-\ell, W)$. The fact that $u$ starts with a segment of slope $\theta-\theta_{m}$ can be proved exactly in the same way.

Let us start by fixing a small number $\alpha>0$. We will focus on the interval $(W-\alpha, W)$, and we will eventually prove that $\arctan \left(u^{\prime}\right) \equiv-\theta_{m}$ on this interval as soon as $\alpha$ is small enough, depending only on the data of the problem (i.e., $\theta, \theta_{m}, \mathcal{N}$ and $m$ ). We will argue in two steps. Step I. The slope of u cannot be "too small" near $W$.

In this first step, we will show that $\arctan \left(u^{\prime}\right) \geq-\theta_{m}$ in $(W-\alpha, W)$ if $\alpha \leq \ell$, where $\ell$ can be bounded from below only in terms of $m$. In particular, $\arctan \left(u^{\prime}\right) \geq-\theta_{m}$ in $\left.(W-\ell, W)\right)$. To show this fact, set

$$
B:=\left\{x \in(W-\alpha, W): \arctan \left(u^{\prime}(x)\right)<-\theta_{m}\right\}, \quad \tau:=|B| .
$$

Define $\tilde{u}:[0, W+\beta] \rightarrow \mathbb{R}$ to be the modified function shown in Figure 8 . We let $\tilde{u}=u$ on $[0, W-\alpha]$, and we define $\tilde{u}^{\prime}=u^{\prime}$ in $(W-\alpha, W) \backslash B$, and $\tilde{u}^{\prime}=\tan \left(-\theta_{m}\right)$ on $B$ and on $[W, W+\beta]$, where $\beta \geq 0$ is such that $\tilde{u}(W+\beta)=0$. Notice the following immediate geometric estimates,

$$
\beta=O(\tau), \quad \max _{x \in(W-\alpha, W+\beta)}\{\tilde{u}(x)-u(x)\}=O(\tau), \quad|| S(u)|-| S(\tilde{u})||=O(\alpha \tau) .
$$

Consider now first the surface energy. Since $u$, being an energy minimizer, must belong to $\mathcal{A}$

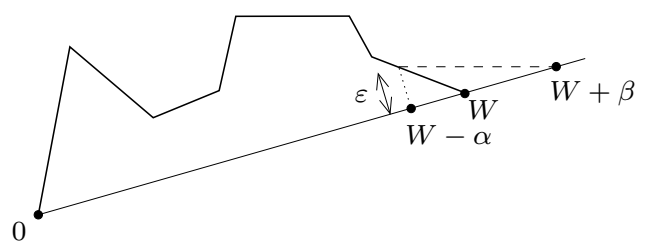

Figure 8. Comparison function with a horizontal segment at the end.

by Theorem A, we have

$$
E_{\mathrm{S}}(\tilde{u}) \leq E_{\mathrm{S}}(u)-c \tau
$$

for some geometric constant $c>0$, depending only on $\theta, \theta_{m}$ and $\mathcal{N}$.

Let us then consider the elastic energy. For every $y \in(0, W-\alpha)$, we set

$$
\varphi(y):=\int_{W-\alpha}^{W+\beta}\left(\tilde{u}^{\prime}(x)-u^{\prime}(x)\right) \ln (x-y) d x,
$$

where $u$ is extended by 0 in $(W, W+\beta)$. Since, by definition, the function $\tilde{u}-u$ is positive in $(W-\alpha, W+\beta)$, but its value is 0 at both the extremes of the interval, then again by the killing 
Lemma we find that $\varphi<0$ and $\varphi^{\prime}<0$. We can now write

$$
\begin{aligned}
E_{\mathrm{r}}(\tilde{u})-E_{\mathrm{r}}(u)=2 \int_{0}^{W-\alpha} & u^{\prime}(y) \varphi(y) d y \\
& +\int_{W-\alpha}^{W+\beta} \int_{W-\alpha}^{W+\beta}\left(\tilde{u}^{\prime}(y) \tilde{u}^{\prime}(x)-u^{\prime}(y) u^{\prime}(x)\right) \ln (|y-x|) d x d y
\end{aligned}
$$

and estimate the two terms separately. Concerning the first one, since $\varphi<0$ we have

$$
\int_{0}^{W-\alpha} u^{\prime}(y) \varphi(y) d y \leq-K \int_{0}^{W-\alpha} \varphi(y) d y
$$

and, in turn, for every $y \in(0, W-\alpha)$, by the definition of $\tilde{u}$ and recalling (6.1) we get

$$
\begin{aligned}
\varphi(y) & =\int_{W-\alpha}^{W}\left(\tilde{u}^{\prime}(x)-u^{\prime}(x)\right) \ln (x-y) d x+\int_{W}^{W+\beta}\left(\tilde{u}^{\prime}(x)-u^{\prime}(x)\right) \ln (x-y) d x \\
& \geq \ln (W-\alpha-y) \int_{W-\alpha}^{W} \tilde{u}^{\prime}(x)-u^{\prime}(x) d x+\ln (W+\beta-y) \int_{W}^{W+\beta} \tilde{u}^{\prime}(x)-u^{\prime}(x) d x \\
& =\max _{x \in(W-\alpha, W+\beta)}\{\tilde{u}(x)-u(x)\}(\ln (W-\alpha-y)-\ln (W+\beta-y)) \\
& \geq-O(\tau) \ln \left(1+\frac{\alpha+\beta}{W-\alpha-y}\right) .
\end{aligned}
$$

Inserting this estimate into (6.4) we get

$$
\int_{0}^{W-\alpha} u^{\prime}(y) \varphi(y) d y \leq O(\tau) \int_{0}^{W-\alpha} \ln \left(1+\frac{\alpha+\beta}{W-\alpha-y}\right) d y=O(\tau) \int_{0}^{W-\alpha} \ln \left(1+\frac{\alpha+\beta}{\sigma}\right) d \sigma .
$$

Now, also recalling that $\beta=O(\tau)$ and that $\tau \leq \alpha$, on the one hand we have

$$
\int_{0}^{\alpha+\beta} \ln \left(1+\frac{\alpha+\beta}{\sigma}\right) d \sigma=(\alpha+\beta) \int_{0}^{1} \ln \left(1+\frac{1}{\sigma}\right) d \sigma=O(\alpha)
$$

and on the other hand we have

$$
\int_{\alpha+\beta}^{W-\alpha} \ln \left(1+\frac{\alpha+\beta}{\sigma}\right) d \sigma \leq \int_{\alpha+\beta}^{W-\alpha} \frac{\alpha+\beta}{\sigma} d \sigma=O(\alpha|\ln (\alpha)|),
$$

from which we finally deduce that

$$
\int_{0}^{W-\alpha} u^{\prime}(y) \varphi(y) d y \leq O(\alpha|\ln (\alpha)| \tau) .
$$

Concerning the second term in (6.3), we have

$$
\begin{aligned}
\mid \int_{W-\alpha}^{W+\beta} & \int_{W-\alpha}^{W+\beta}\left(\tilde{u}^{\prime}(y) \tilde{u}^{\prime}(x)-u^{\prime}(y) u^{\prime}(x)\right) \ln (|y-x|) d x d y \mid \\
& \leq K \int_{B} \int_{W-\alpha}^{W+\beta}|\ln (|x-y|)| d x d y \leq 2 K \tau \int_{0}^{\alpha+\beta}|\ln (t)| d t=O(\alpha|\ln (\alpha)| \tau) .
\end{aligned}
$$

Inserting (6.5) and (6.6) into (6.3), we get

$$
E_{\mathrm{r}}(\tilde{u})-E_{\mathrm{r}}(u) \leq O(\alpha|\ln (\alpha)| \tau) .
$$


We are now in position to conclude. Recalling the formulas of Section 5 for the energy of the rescaled profiles, putting together (6.1), (6.2) and (6.7) we obtain that the rescaled profile $v$ of $\tilde{u}$ having mass $|S(v)|=|S(u)|=m$ has energy

$$
E(v) \leq E(u)+O(\alpha|\ln (\alpha)| \tau)-c \tau<E(u),
$$

where the last inequality (which gives a contradiction because $u$ is an energy minimizer of mass $m$ ) is true as soon as $\alpha$ is small enough and if $\tau>0$. We derive that, whenever $\alpha \leq \ell$ for some length $\ell=\ell\left(m, \theta, \theta_{m}, \mathcal{N}\right)$, it must be $\tau=0$; in other words, any energy minimizing profile has slope $u^{\prime}(x) \geq-\tan \left(\theta_{m}\right)$ for a.e. $x \in(W-\ell, W)$.

Step II. The slope of u cannot be "too big" near $W$.

In this second step we will show that, if $\alpha \leq \ell$ for a suitable $\ell=\ell\left(m, \theta, \theta_{m}, \mathcal{N}\right)$, then it must be $u^{\prime} \leq 0$ in $(W-\alpha, W)$. Since $u$ is an energy minimizer, thus $u \in \mathcal{A}$ by Theorem A, this information together with that of Step I ensure that $u^{\prime} \equiv \tan \left(\theta_{m}\right)$ in $(W-\ell, W)$, hence that any optimal profile ends with a segment of slope $-\theta_{m}$. Since proving that any optimal profile starts with a segment of slope $\theta-\theta_{m}$ is completely equivalent, after this step the proof of the theorem will be concluded.

The argument of this second step is actually almost identical to the one of the first step. We define this time

$$
B:=\left\{x \in(W-\alpha, W): u^{\prime}(x)>0\right\}, \quad \tau:=|B| \geq 0,
$$

and we let $\tilde{u}:[0, W-\beta] \rightarrow \mathbb{R}$ be the modified function satisfying $\tilde{u}=u$ in $[0, W-\alpha], \tilde{u}^{\prime} \equiv$ $-\tan \left(\theta_{m}\right)$ on $(W-\alpha, W-\beta)$, and $\tilde{u}(W-\beta)=0$. It follows that

$$
\beta=O(\tau), \quad \max _{x \in(W-\alpha, W-\beta)}\{u(x)-\tilde{u}(x)\}=O(\tau), \quad|| S(u)|-| S(\tilde{u})||=O(\alpha \tau),
$$

and this time, since $\tilde{u}-u$ is negative in $(W-\alpha, W)$ but null at the extremes of this interval, by letting

$$
\varphi(y):=\int_{W-\alpha}^{W}\left(\tilde{u}^{\prime}(x)-u^{\prime}(x)\right) \ln (x-y) d x
$$

one has by the killing Lemma that $\varphi>0$ and $\varphi^{\prime}>0$ (this time we are extending $\tilde{u}$ to 0 in $(W-\beta, W))$. Exactly as in $(6.3)$ we can estimate

$$
\begin{aligned}
E_{\mathrm{r}}(\tilde{u})-E_{\mathrm{r}}(u)=2 \int_{0}^{W-\alpha} & u^{\prime}(y) \varphi(y) d y \\
& +\int_{W-\alpha}^{W} \int_{W-\alpha}^{W}\left(\tilde{u}^{\prime}(y) \tilde{u}^{\prime}(x)-u^{\prime}(y) u^{\prime}(x)\right) \ln (|y-x|) d x d y,
\end{aligned}
$$

and the very same calculations which led to (6.5) and (6.6) also in this case ensure that

$$
E_{\mathrm{r}}(\tilde{u})-E_{\mathrm{r}}(u) \leq O(\alpha|\ln (\alpha)| \tau)
$$

and we conclude exactly as in the first step. 


\section{Theorem C: The Minimizers for SMALl volumes ARE HALF-PyRAmids}

Our last result is that, as already observed with experiments and numerical simulations (see $[18,19]$ ), if the mass is small enough then the energy minimizers are half-pyramids . As explained in the introduction (see Figure 2), the half-pyramids are the simplest possible profiles, namely, profiles which consist of just two segments, a first one with the smallest possible positive slope (that is, $\arctan \left(\theta-\theta_{m}\right)$ ) and a second one with the biggest possible negative slope (that is, $\left.\arctan \left(-\theta_{m}\right)\right)$. Clearly, for every positive number $m$ there exists exactly one half-pyramid with mass $m$.

The half-pyramid is always the unique minimizer of the surface energy (see also the proof of Theorem $\mathrm{C}$ below). Consequently, since the different scaling properties of surface and elastic energy imply that the effect of the surface energy dominates the one of the elastic energy for volumes which converge to 0 , it is intuitive that, when the volume goes to 0 , the solutions tend to be closer and closer to the half-pyramids. What our result says, instead, is quite stronger: namely, that the half-pyramid is the only solution as soon as the volume is smaller than a critical threshold.

Theorem C (Half-pyramids are minimizers for small mass). There exists some $\bar{m}>0$ such that, for every $m<\bar{m}$, the half-pyramid is the unique energy minimizer with mass $m$.

Proof. We divide the proof in a few steps.

Step I. An elastic energy estimate for "close profiles".

In this first step we consider two functions which coincide outside of a small interval, and we give an upper bound for the difference of the elastic energies. Precisely, consider two profiles $u, \tilde{u} \in \mathcal{A}$ with $u:[0, W] \rightarrow \mathbb{R}$ and $\tilde{u}:\left[0, W^{\prime}\right] \rightarrow \mathbb{R}$, with the property that there exists an interval $(a, b) \in(0, W)$ such that, setting $\delta:=W^{\prime}-W$ and $b^{\prime}:=b+\delta \geq a$, the following holds,

$$
\tilde{u}(x)=u(x) \quad \text { for } 0 \leq x \leq a, \quad \tilde{u}(x)=u(x-\delta) \quad \text { for } b^{\prime} \leq x \leq W^{\prime} .
$$

Figure 9 depicts the simple situation we are considering. The goal of this step is to show that for every $\kappa>0$ there exist two positive constants $\bar{W}$ and $\bar{\varepsilon}$ such that

$$
\left\{\begin{array}{c}
W \leq \bar{W}, \\
\max \left\{b, b^{\prime}\right\}-a=: \varepsilon \leq \bar{\varepsilon},
\end{array} \Longrightarrow\left|E_{\mathrm{r}}(\tilde{u})-E_{\mathrm{r}}(u)\right| \leq \kappa \varepsilon .\right.
$$

The proof of (7.1) comes from calculations, which are similar to those already undertaken in
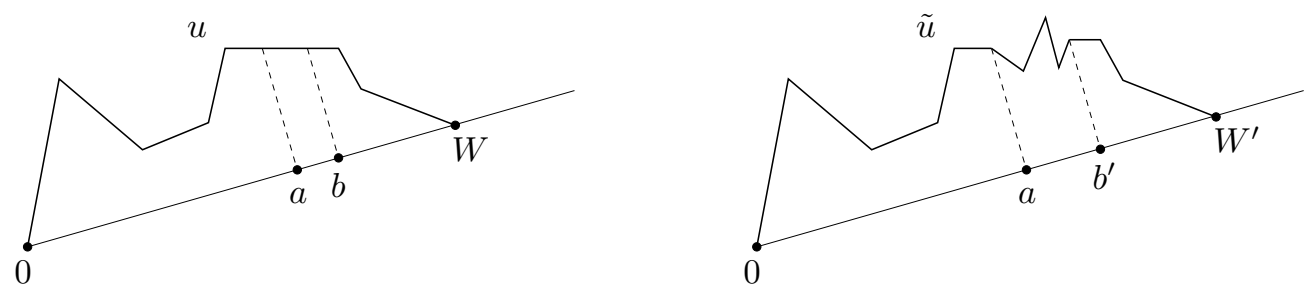

Figure 9. The functions $u$ and $\tilde{u}$ in Step I. 
the last sections. A first, rough but useful, estimate says that

$$
\begin{aligned}
\left|E_{\mathrm{r}}(\tilde{u})-E_{\mathrm{r}}(u)\right| \leq \int_{a}^{b} \int_{0}^{W} \mid u^{\prime}(x) u^{\prime}(y) & \ln (|y-x|)\left|+\int_{a}^{b^{\prime}} \int_{0}^{W^{\prime}}\right| \tilde{u}^{\prime}(x) \tilde{u}^{\prime}(y) \ln (|y-x|) \mid \\
& +\int_{0}^{a} \int_{b}^{W}\left|u^{\prime}(x) u^{\prime}(y)(\ln (x-y+\delta)-\ln (x-y))\right| .
\end{aligned}
$$

Concerning the first two terms, we have that

$$
\int_{a}^{b} \int_{0}^{W}\left|u^{\prime}(x) u^{\prime}(y) \ln (|y-x|)\right| \leq K(b-a) \int_{0}^{W}|\ln (t)| d t \leq c(W) \varepsilon,
$$

where $c(W)$ is a constant depending only on $\theta, \theta_{m}, \mathcal{N}$ and $W$, and which goes to 0 when $W$ goes to 0 . An analogous estimate holds also for the second term in (7.2). Concerning the third term, we observe that

$$
\begin{gathered}
\int_{0}^{a} \int_{b}^{W}\left|u^{\prime}(x) u^{\prime}(y)(\ln (x-y+\delta)-\ln (x-y))\right| \leq K \int_{0}^{W} \int_{0}^{W} \ln \left(1+\frac{\varepsilon}{s+t}\right) d s d t \\
\leq K \varepsilon \int_{0}^{W} \int_{0}^{W} \frac{1}{s+t}=K \varepsilon \int_{0}^{W} \ln \left(1+\frac{W}{t}\right)=K \varepsilon W \int_{0}^{1} \ln \left(1+\frac{1}{t}\right) \leq c(W) \varepsilon
\end{gathered}
$$

where again $c(W)$ is a constant which goes to 0 when $W \rightarrow 0$. The last two estimates, together with (7.2), prove the validity of (7.1).

Step II. The only possible slopes are $\theta-\theta_{m}$ and $-\theta_{m}$.

In this second step we show that, if $m$ is small enough then every energy minimizer of mass $m$ can only have slopes $\theta-\theta_{m}$ and $-\theta_{m}$. We will only prove that an energy minimizer $u$ with small mass must satisfy $u^{\prime} \leq \arctan \left(\theta-\theta_{m}\right)$ almost everywhere, because the proof that $u^{\prime} \geq \arctan \left(-\theta_{m}\right)$ almost everywhere is identical. Let $u$ be such a minimizer, and assume by contradiction that there are two points $a, b \in(0, W)$ such that

$$
\frac{u(b)-u(a)}{b-a} \geq \arctan \left(\frac{3}{2} \theta-\theta_{m}\right) .
$$

Notice that the existence of such a pair of points is equivalent to the negation of the claim, and that, thanks to Theorem A, $\frac{3}{2}$ could be replaced by any number strictly smaller than 2 . Let us now consider the modification $\tilde{u}$ shown in Figure 10 (left): let $b^{\prime}$ be the number such that

$$
\frac{u(b)-u(a)}{b^{\prime}-a}=\arctan \left(\theta-\theta_{m}\right)
$$

and let $\tilde{u}$ be the continuous function which equals $u$ in $(0, a)$, whose slope is constantly $\theta-\theta_{m}$ in $\left(a, b^{\prime}\right)$, and which coincides with the translated copy of $u_{\mid(b, W)}$ on $\left(b^{\prime}, W^{\prime}\right)=\left(b^{\prime}, W+b^{\prime}-b\right)$. If we set $\varepsilon:=b^{\prime}-a>b-a$, then the usual perimeter estimate yields

$$
E_{\mathrm{S}}(\tilde{u}) \leq E_{\mathrm{S}}(u)-2 \kappa \varepsilon
$$

for a purely geometric constant $\kappa>0$. Applying Step I, we find two positive constants $\bar{W}$ and $\bar{\varepsilon}$ such that (7.1) holds. Notice now that, by Proposition 2.4 and Corollary 2.6, $W \leq \bar{W}$ holds provided that $m$ is small enough. Concerning the fact that $\varepsilon \leq \bar{\varepsilon}$, this also holds provided that the points $a$ and $b$ satisfying (7.3) are chosen sufficiently close to each other, which is of course possible. As a consequence, (7.1) implies that $E(\tilde{u}) \leq E(u)-\kappa \varepsilon$. Finally, observe that 
||$S(\tilde{u})|-| S(u)||$ is of order $\varepsilon^{2}$, and so the estimates of Section 5 ensure that the rescaled copy of $\tilde{u}$ having the same mass as $u$ has a strictly less total energy. The contradiction proves the claim of this second step.
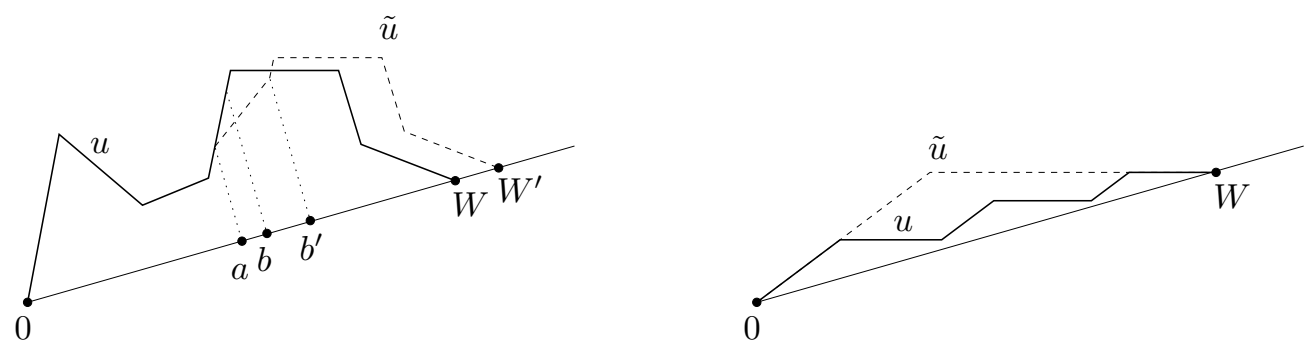

Figure 10. The variations in Steps II and III.

\section{Step III. The solution is a half-pyramid.}

In view of the previous step, we know that an energy minimizer $u$ of small mass can only have the two "most horizontal" slopes, namely $-\theta_{m}$ and $\theta-\theta_{m}$. However, this still does not imply that $u$ is the half-pyramid. Let then $u$ be any energy minimizer, and write $(0, W)=A \cup B$ with $A:=\left\{\tan u^{\prime}=\theta-\theta_{m}\right\}$ and $B:=\left\{\tan u^{\prime}=-\theta_{m}\right\}$. Define $\tilde{u}$ to be the half-pyramid having total width equal to $W$, as in Figure 10 (right). Observe that, by definition, the two sides of the half-pyramid have exactly lengths $|A|$ and $|B|$. We claim that

$$
E_{\mathrm{S}}(\tilde{u})=E_{\mathrm{S}}(u), \quad E_{\mathrm{r}}(\tilde{u}) \leq E_{\mathrm{r}}(u), \quad|S(\tilde{u})| \geq|S(u)|,
$$

where the second and the third inequalities are strict unless $u=\tilde{u}$. Since the first and the third property are clearly true, we only have to prove the second one. The idea is that, once the lengths of the sets $A$ and $B$ are given, in order to minimize the elastic energy it is convenient to have the pairs of points with the same derivative as close as possible, and the pairs of points having different derivative as far as possible. Precisely, notice that for every $0 \leq t \leq|A|+|B|$

$$
\left|\left\{(x, y):|y-x| \leq t, \tilde{u}^{\prime}(x)=\tilde{u}^{\prime}(y)\right\}\right| \geq\left|\left\{(x, y):|y-x| \leq t, u^{\prime}(x)=u^{\prime}(y)\right\}\right|,
$$

where at least one of the inequalities is strict unless $u=\tilde{u}$. Hence, the second property in (7.4) is immediate taking into consideration that the logarithm is an increasing function and that

$$
\begin{aligned}
\int_{0}^{W} \int_{0}^{W} & \left.u^{\prime}(y) u^{\prime}(x) \ln (|y-x|) d x d y=\arctan ^{2}\left(\theta-\theta_{m}\right) \int_{0}^{W} \ln (t) \mid\left\{(x, y) \in A^{2}:|y-x|=t\right)\right\} \mid \\
& \left.+\arctan ^{2}\left(\theta_{m}\right) \int_{0}^{W} \ln (t) \mid\left\{(x, y) \in B^{2}:|y-x|=t\right)\right\} \mid \\
& \left.-2 \arctan \left(\theta-\theta_{m}\right) \arctan \left(\theta_{m}\right) \int_{0}^{W} \ln (t) \mid\{(x, y) \in A \times B:|y-x|=t)\right\} \mid .
\end{aligned}
$$

Having (7.4) established, we conclude the proof. Indeed, assuming that $u \neq \tilde{u}$, we let $\varepsilon<0$ be such that

$$
|S(u)|=(1+\varepsilon)^{2}|S(\tilde{u})|
$$


and define $\hat{u}$ to be the rescaled copy of $\tilde{u}$ having mass $|S(u)|$. With the notation of Section 5 , one has $\hat{u}=\tilde{u}_{\varepsilon}, \hat{u}$ is the half-pyramid of mass $|S(u)|$. Applying now (5.2) and using (7.4), we have

$$
\begin{aligned}
E(\hat{u}) & =(1+\varepsilon)^{2} E_{\mathrm{r}}(\tilde{u})+(1+\varepsilon) E_{\mathrm{S}}(\tilde{u}) \leq(1+\varepsilon)^{2} E_{\mathrm{r}}(u)+(1+\varepsilon) E_{\mathrm{S}}(u) \\
& =E(u)+\varepsilon\left(E_{\mathrm{S}}(u)+2 E_{\mathrm{r}}(u)+\varepsilon E_{\mathrm{r}}(u)\right) .
\end{aligned}
$$

Recalling that $\varepsilon<0$, we find the contradiction $E(\hat{u})<E(u)$ since $E_{\mathrm{S}}(u) \gg\left|E_{\mathrm{r}}(u)\right|$ if $m \ll 1$. Finally, since as already observed one has $E_{\mathrm{S}}(u) \geq c W$ for some positive constant $c$, the claim follows from Step I because $\left|E_{\mathrm{r}}(\tilde{u})\right| \ll W$ provided $m$ (and thus $W$ ) is small enough.

Acknowledgements. The authors warmly thank Brian Spencer for helpful discussions on the subject of this paper, and the Center for Nonlinear Analysis (NSF Grant No. DMS-0635983) where part of this research was carried out. The research of I. Fonseca and of B. Zwicknagl was partially funded by the National Science Foundation under Grant No. DMS-0905778, while the research of A. Pratelli was partially funded by the European Research Council under the Starting Grant No. 258685. A. Pratelli and B. Zwicknagl thank the University of Pavia and the University of Erlangen for their hospitality.

\section{REFERENCES}

[1] P. Bella, M. Goldman, and B. Zwicknagl. Study of island formation on unbounded domains. arXiv:1402.3955, 2014.

[2] E. Bonnetier and A. Chambolle. Computing the equilibrium configuration of epitaxially strained crystalline islands. SIAM J. Appl. Math., 62 (4):1093-1121, 2007.

[3] A. Chambolle and C. J. Larsen. $C^{\infty}$ regularity of the free boundary for a two-dimensional optimal compliance problem. Calc. Var. PDE, 18(1):77-94, 2003.

[4] A. Chambolle and M. Solci. Interaction of a bulk and a surface energy with a geometrical constraint. SIAM J. Math. Anal., 39:77-102, 2007.

[5] U. Denker, A. Rastelli, M. Stoffel, J. Tersoff, G. Katsaros, G. Constantini, K. Kern, N.Y. Jin-Phillip, D. E. Jesson, and O. G. Schmidt. Lateral motion of sige islands driven by surface-mediated alloying. Phys. Rev. Lett., 94:196104, 205.

[6] I. Fonseca, N. Fusco, G. Leoni, and V. Millot. Material voids in elastic solids with anisotropic surface energies. J. Math. Pures Appl., 96(6):591-639, 2011.

[7] I. Fonseca, N. Fusco, G. Leoni, and M. Morini. Equilibrium configurations of epitaxially strained crystaline films: Existence and regularity results. Arch. Rat. Mech. Anal., 186(3):477-537, 2007.

[8] I. Fonseca, N. Fusco, G. Leoni, and M. Morini. Motion of elastic thin films by anisotropic surface diffusion with curvature regularization. Arch. Rat. Mech. Anal., pages 1-42, 2012.

[9] N. Fusco and M. Morini. Equilibrium configurations of epitaxially strained elastic films: Second order minimality conditions and qualitative properties of solutions. Arch. Rat. Mech. Anal., 203:247-327, 2012.

[10] H. Gao and W. D. Nix. Surface roughening of heteroepitaxial thin films. Annual Review of Materials Science, 29:173-209, 1999.

[11] M. Goldman and B. Zwicknagl. Scaling law and reduced models for epitaxially strained crystalline films. SIAM J. Math. Anal., 46:1-24, 2014.

[12] J. L. Gray, R. Hull, and J. A. Floro. Formation of one-dimensional surface grooves from pit instabilities in annealed SiG/Si (100) epitaxial films. Appl. Phys. Lett., 85(15), 2004. 
[13] P. Piovano. Evolution of elastic thin films with curvature regularization via minimizing movements. to appear in Calc. Var. PDE, 2012.

[14] F. M. Ross, R. M. Tromp, and M. C. Reuter. Transition states between pyramids and domes during Ge/Si island growth. Science, 286:1931, 1999.

[15] T. P. Schulze and P. Smereka. Kinetic monte carlo simulation of heteroepitaxial growth: Wetting layers, quantum dots, capping, and nanorings. Phys. Rev. B, 86:235313, Dec 2012.

[16] B. J. Spencer. Asymptotic derivation of the glued-wetting-layer model and contact-angle condition for Stranski-Krastanow islands. Phys. Rev. B, 59:2011-2017, 1999.

[17] B. J. Spencer and J. Tersoff. Equilibrium shapes and properties of epitaxially strained islands. Phys. Rev. Lett., 79:4858-4861, 1997.

[18] B. J. Spencer and J. Tersoff. Asymmetry and shape transitions of epitaxially strained islands on vicinal surfaces. Appl. Phys. Lett., 96:073114, 2010.

[19] B. J. Spencer and J. Tersoff. Symmetry breaking in shape transitions of epitaxial quantum dots. Phys. Rev. $B, 87: 161301$ (5 pages), 2013.

[20] J. Stangl, V. Holý, and G. Bauer. Structural properties of self-organized semiconductor nanostructures. Rev. Mod. Phys., 76:725, 2004.

[21] P. Sutter, E. Sutter, and L. Vescan. Barrierless self-assembly of Ge quantum dots on Si(001) substrates with high local vicinality. Appl. Phys. Lett., 87:161916, 2005.

[22] J. Tersoff and R. M. Tromp. Shape transition in growth of strained islands: Spontaneous formation of quantum wires. Phys. Rev. Lett., 70(18):2782-2786, 1993. 